\title{
EUCLIDEAN UPGRADING FROM SEGMENT LENGTHS
}

\author{
JOSÉ I. RONDA* AND ANTONIO VALDÉS**
}

\begin{abstract}
We address the problem of the recovery of Euclidean structure of a projectively distorted $n$-dimensional space from the knowledge of segment lengths. This problem is relevant, in particular, for Euclidean reconstruction with uncalibrated cameras, extending previously known results in the affine setting. The key concept is the Quadric of Segments (QoS), defined in a higher-dimensional space by the set of segments of a fixed length from which Euclidean structure can be obtained in closed form. We have intended to make a thorough study of the properties of the QoS, including the determination of the minimum number of segments of arbitrary length that determine it and its relationship with the standard geometric objects associated to the Euclidean structure of space. Explicit formulas are given to obtain the dual absolute quadric and the absolute quadratic complex from the QoS. Experiments with real and synthetic images evaluate the performance of the techniques.
\end{abstract}

* Grupo de Tratamiento de Imágenes Universidad Politécnica de Madrid 28040 Madrid, Spain

jir@gti.ssr.upm.es
** Dep. de Geometría y Topología Universidad Complutense de Madrid 28040 Madrid, Spain

Antonio_Valdes@mat.ucm.es

\section{INTRODUCTION}

A standard strategy for 3D reconstruction from images when the camera internal parameters are unknown starts by computing a projective reconstruction of the scene, i.e., a 3D reconstruction distorted by an unknown spatial homography. This step must be followed by a Euclidean upgrading, for which some additional data are required. Therefore, the problem of recovery of Euclidean structure of a projectively distorted space has deserved considerable attention $[2,6-8,10-12,19,21]$.

Euclidean upgrading can be obtained from the geometric properties of either physical objects or geometrical entities identified in the scene. Autocalibration methods rely on geometric objects that can be determined when the cameras satisfy certain restrictions. For instance, each camera with known principal point and skew provides three pairwise orthogonal planes that can be used to compute the Euclidean structure of space [18], or each camera with known skew and aspect ratio contributes with two lines through the optical center intersecting the absolute conic that can be used with the same purpose [12].

Other approaches to 3D reconstruction from images make first use of different calibration objects to compute the intrinsic and extrinsic parameters of the cameras before obtaining the reconstruction. The most classical method to calibrate cameras makes use a calibration pattern [22], i.e., an object for which the 3D coordinates of some points are known with precision with respect to a reference system attached to the object. Different techniques trying to relax the imposed conditions to the 3D object appeared such as Zhang's calibration method [27], in which only two different views of a planar checkboard

Date: February 23, 2010.

This work has been partly supported by the Comisión Interministerial de Ciencia y Tecnología (CICYT) of the Spanish Government. 
are needed to compute the intrinsic parameters of the camera. Other alternatives make use of a moving stick with three marked points with known relative positions, one of them fixed [28], or of three orthogonal marked sticks [14]. Spheres [1,26] or revolution surfaces [25] have also been proposed.

In this paper we assume that the lengths of a set of segments in the scene are known. One particular relevant instance is that of a camera recording one or more moving segments of constant length. This is a situation of practical interest, for example in scenes in which human body segments appear [20]. Up to our knowledge, this problem has not been studied in its full generality. In [9] the analogous problem when an affine reconstruction is previously known was solved. Here we only assume a projective reconstruction and show how the Euclidean geometry can be completely recovered just from the knowledge of segment lengths. This problem is more involved, even in the two-dimensional case, due to the high non-linearity of the equations relating the circular points at infinity with the metric [15, Eq. 22].

We have chosen to make a general $n$-dimensional treatment, thus unifying the cases $n=$ 2,3 . We first introduce a convenient geometric space to represent segments, identifying a segment with a degenerated dual quadric of rank $\leq 2$. Therefore segments appear as an algebraic variety of $\mathbf{P}^{N}, N=(n+1)(n+2) / 2-1$. We show that segments of fixed length $d$ are determined by a unique quadric $\mathrm{C}$ of $\mathbf{P}^{N}$, which we call the quadric of segments (QoS) which naturally decomposes as a sum $\mathrm{C}=\mathrm{C}_{1}+\frac{d^{2}}{2} \mathrm{C}_{2}$, where the quadric $\mathrm{C}_{2}$ encodes the affine geometry of space while $\mathrm{C}_{1}$ determines Euclidean geometry up to a scale factor. The pair $\left(\mathrm{C}_{1}, \mathrm{C}_{2}\right)$ determines also the scale up to a sign, i.e., Euclidean geometry up to mirror symmetry.

The space of quadrics of $\mathbf{P}^{N}$ is seen to decompose into two orthogonal subspaces, one spanned by the possible matrices $\mathrm{C}_{1}$ and the other by the possible matrices $\mathrm{C}_{2}$. This decomposition allows to optimize the number of segments needed to compute the QoS in the linear algorithm proposed so that $(N+1)(N+2) / 2-1$ segments of known length suffice.

We provide explicit formulas to recover the hyperplane at infinity from $\mathrm{C}_{2}$ and to recover the dual absolute quadric $\mathrm{Q}_{\infty}^{*}$ from $\mathrm{C}_{1}$. We end the theoretical contributions of the paper studying the relationship between the QoS and the Absolute Quadratic Complex (AQC) $[13,16,23]$, showing that $\mathrm{C}_{1}$ and the AQC are essentially equivalent objects.

We conclude the paper providing experimental results, both for real and synthetic images evaluating the performance of the proposed techniques and showing the feasibility of the approach.

\section{BACKGROUND AND NOTATIONS}

We assume that cameras are modeled [4] by the equation $\mathbf{q} \sim P \mathbf{Q}$, where $\mathbf{Q}=$ $(x, y, z, t)^{T}$ denotes the Euclidean homogeneous coordinates of a spatial point, $\mathbf{q}=$ $(u, v, w)^{T}$ denotes the homogeneous coordinates of an image point, and $P$ is a $3 \times 4$ matrix.

We recall here [4] that it is possible to obtain a projective calibration only from image point correspondences. This means that, given a set of projected points $\mathbf{q}_{i j}$ obtained with $N$ cameras, $N \geq 2$, we can obtain a set of matrices $\hat{P}_{i}$ and a set of point coordinates $\hat{\mathbf{Q}}_{j}$ such that $\mathbf{q}_{i j} \sim \hat{\hat{P}}_{i} \hat{\mathbf{Q}}_{j}$, where $\hat{P}_{i}=P_{i} H^{-1}$ and $\hat{\mathbf{Q}}_{j}=H \mathbf{Q}_{j}$ for some non-singular $4 \times 4$ matrix $H$. 
Euclidean calibration can be defined as the obtainment of a matrix $H$ changing the projective coordinates of a given projective calibration to some Euclidean coordinate system. The aim of this paper is to obtain the Euclidean calibration from the knowledge of segment lengths. It is well-known that Euclidean calibration up to a scale factor is equivalent to the recovery of the absolute conic at infinity $\Omega_{\infty}$ or any of its geometric equivalencies, such as the dual absolute quadric $Q_{\infty}[21]$ or the absolute quadratic complex $\Sigma[13,16,23]$.

Analogous to Euclidean calibration is the notion of affine calibration. In this case the structure of the scene is known up to an affine 3D transformation. Affine calibrations are obtained in particular when we have affine cameras [4, p. 173].

Let us suppose that we know the lengths $d_{i}$ of a set of segments $\left\{\mathbf{X}_{i}, \mathbf{Y}_{i}\right\}$ in a projective reconstruction of space with no other particular structure. We would like to determine an Euclidean upgrading from this knowledge. This problem has been solved in the affine case computing an affine adjustment consisting in the determination of an affinity transforming the set of segments so that their lengths become as close as possible, in the least squares sense, to the given values [9]. An affinity matrix $U$ is obtained from the matrix $\mathrm{U}^{\top} \mathrm{U}$ obtained by solving the least squares problem using a Cholesky factorization.

\section{Geometric representation of Segments}

Given a segment $\{\mathbf{x}, \mathbf{y}\}$ we define the symmetric matrix

$$
\mathbf{S}=\mathbf{S}(\mathbf{x}, \mathbf{y})=\mathbf{x y}^{\top}+\mathbf{y} \mathbf{x}^{\top}=\left(x_{i} y_{j}+x_{j} y_{i}\right)=\left(z_{i j}\right) .
$$

The matrix $\mathrm{S}$ can be interpreted as the dual quadric given by all the hyperplanes passing either through $\mathbf{x}$ or $\mathbf{y}$. Its rank is two whenever $\mathbf{x}$ and $\mathbf{y}$ are different points, being otherwise one. Note that this construction is the symmetric counterpart of the Segre embedding for the representation of ordered point pairs [3, p. 25].

Result 3.1. The mapping $\{\mathbf{x}, \mathbf{y}\} \mapsto \mathrm{S}(\mathbf{x}, \mathbf{y})$ identifies non-oriented segments with $(n+$ $1) \times(n+1)$ projective symmetric matrices of rank $\leq 2$.

Proof. It is enough to define the inverse mapping of $\{\mathbf{x}, \mathbf{y}\} \mapsto \mathbf{S}(\mathbf{x}, \mathbf{y})$, so let $\mathbf{S}$ be a ranktwo symmetric matrix and let us check that there exists unique $\mathbf{x}, \mathbf{y}$ such that $\mathbf{S}=\mathbf{S}(\mathbf{x}, \mathbf{y})$. We suppose that rank $\mathbf{S}(\mathbf{x}, \mathbf{y})=2$ being the rank one case trivial. Being $\mathbf{S}$ rank two and symmetric, there exists a change of coordinates $\mathrm{H}$ such that $\mathrm{H}^{\top} \mathrm{SH}=\operatorname{diag}(1,1,0, \ldots, 0)$. We set $\mathbf{x}^{0}, \mathbf{y}^{0}=(1, \pm i, 0, \ldots, 0)^{\top}$. It is immediate to check that $\mathbf{x}=\mathrm{Hx}_{0}, \mathbf{y}=\mathrm{Hy}_{0}$ are the required vectors. Regarding its uniqueness, first we note that $\mathbf{x}_{0}, \mathbf{y}_{0}$ are, up to scale, the only vectors such that $\mathbf{x}^{0} \mathbf{y}^{0^{\top}}+\mathbf{y}^{0} \mathbf{x}^{0^{\top}} \sim \operatorname{diag}(1,1,0, \ldots, 0)$, as can be easily proved. Therefore $\mathbf{x}, \mathbf{y}$ are also unique, since the existence of another pair $\mathbf{x}^{\prime}, \mathbf{y}^{\prime}$ with $\mathbf{S}\left(\mathbf{x}^{\prime}, \mathbf{y}^{\prime}\right)=\mathbf{S}$ would lead to the existence of $\mathbf{x}_{0}^{\prime}=\mathrm{H}^{-1} \mathbf{x}^{\prime}$ and $\mathbf{y}_{0}^{\prime}=\mathrm{H}^{-1} \mathbf{y}^{\prime}$ with $\mathrm{S}\left(\mathbf{x}_{0}^{\prime}, \mathbf{y}_{0}^{\prime}\right)=\mathrm{S}_{0}$.

It will be useful to endow the space of symmetric matrices with the product given by

$$
\langle\mathrm{A}, \mathrm{B}\rangle=\frac{1}{2} \operatorname{trace}(\mathrm{AB}) \text {. }
$$

Using the definition of $\mathrm{S}$ and the equality trace $(\mathrm{AB})=$ trace $(\mathrm{BA})$ it can be easily checked that this scalar product satisfies

$$
\langle\mathrm{S}(\mathbf{u}, \mathbf{v}), \mathrm{S}(\mathbf{w}, \mathbf{z})\rangle=\langle\mathbf{u}, \mathbf{w}\rangle\langle\mathbf{v}, \mathbf{z}\rangle+\langle\mathbf{u}, \mathbf{z}\rangle\langle\mathbf{v}, \mathbf{w}\rangle .
$$


Given a symmetric matrix $\mathrm{A}=\left(z_{i j}\right)$ we define

$$
\nu(\mathrm{A})=\left(\frac{z_{00}}{\sqrt{2}}, \frac{z_{11}}{\sqrt{2}}, \ldots, \frac{z_{n n}}{\sqrt{2}}, z_{01}, z_{02}, \ldots, z_{0 n}, z_{12}, \ldots, z_{1 n}, z_{23}, \ldots, z_{2 n}, \ldots, z_{n-1, n}\right),
$$

so we have a Euclidean coordinate system in the space of symmetric matrices, since it satisfies

$$
\langle\mathrm{A}, \mathrm{B}\rangle=\nu(\mathrm{A})^{\top} \nu(\mathrm{B})=\langle\nu(\mathrm{A}), \nu(\mathrm{B})\rangle .
$$

Finally we define

$$
\sigma(\mathbf{x}, \mathbf{y})=\nu(\mathbf{S}(\mathbf{x}, \mathbf{y}))
$$

thus associating a point of $\mathbf{P}^{N}$ to a segment $\{\mathbf{x}, \mathbf{y}\}$, where

$$
N=\left(\begin{array}{c}
n+1 \\
2
\end{array}\right)-1
$$

\section{The Quadric of Segments of Length $d$.}

The equation of a sphere of center $\mathbf{Y}$ and radius $d \geq 0$

$$
\sum_{i=1}^{n}\left(X_{i}-Y_{i}\right)^{2}-d^{2}=0
$$

can also be interpreted as that of the set of non-oriented segments $\{\mathbf{X}, \mathbf{Y}\}$ at distance $d$. From now on we will use the more convenient homogeneous coordinates $\left(x_{i}\right),\left(y_{i}\right)$, so that with $X_{i}=x_{i} / x_{0}, Y_{j}=y_{j} / y_{0}$ the previous equation becomes

$$
\sum_{i=1}^{n}\left(x_{i} y_{0}-y_{i} x_{0}\right)^{2}-d^{2} x_{0}^{2} y_{0}^{2}=0 .
$$

Remark 4.1. The homogenization of equation (8) introduces new solutions. In fact, if $(\mathbf{x}, \mathbf{y}) \in \mathcal{V}_{d}$ and, say $\mathbf{x}$, lies in the hyperplane at infinity $x_{0}=0$, then either $\mathbf{x}$ lies in the absolute quadric ${ }^{1} \Omega_{\infty}$

$$
\sum_{i=1}^{n} x_{i}^{2}=0, x_{0}=0,
$$

or both $\mathbf{x}$ and $\mathbf{y}$ lie in the plane at infinity.

Remark 4.2. Since we are considering complex coordinates, spheres of radius 0 are not just points but complex cones. In fact, let us suppose that $d=0$ and let $\mathbf{x}$ be a point satisfying equation (9). Then it is easy to check that all the points of the line through $\mathbf{x}$ and $\mathbf{y}$, also satisfy the equation, so it represents a cone of vertex $\mathbf{y}$. The intersection of this cone with the hyperplane at infinity is the absolute quadric.

Since $\mathcal{V}_{d}$ is a symmetric variety of bigrade $(2,2)$, its equation is of the form

$$
\sum a_{i j k l} x_{i} x_{j} y_{k} y_{l}=0
$$

where the coefficients $a_{i j k l}$ satisfy the symmetries $a_{i j k l}=a_{j i k l}=a_{k l i j}$. Since there are $\left(\begin{array}{c}n+1 \\ 2\end{array}\right)$ unordered products $x_{i} x_{j}$ ( or $\left.y_{k} y_{l}\right)$, there is a total of $\left(\begin{array}{c}N+1 \\ 2\end{array}\right)$ independent coefficients up to a common scale factor, where $N$ is given by (7).

\footnotetext{
${ }^{1}$ In dimension three the absolute quadric is usually called the absolute conic at infinite.
} 
A more adequate geometric setting to deal with varieties of this type will permit to treat the problem in a more compact way and to extract efficiently the information encoded by $\mathcal{V}_{d}$. For this purpose we introduce the $N+1$ variables

$$
z_{i j}=x_{i} y_{j}+x_{j} y_{i}, i \leq j
$$

so equation (9) is transformed into

$$
\begin{aligned}
& \sum_{i=1}^{n}\left(x_{i} y_{0}-y_{i} x_{0}\right)^{2}-d^{2} x_{0}^{2} y_{0}^{2} \\
= & \sum_{i=1}^{n}\left(\left(x_{i} y_{0}+y_{i} x_{0}\right)^{2}-4 x_{i} y_{0} y_{i} x_{0}\right)-d^{2} x_{0}^{2} y_{0}^{2} \\
= & \sum_{i=1}^{n}\left(z_{0 i}^{2}-z_{i i} z_{00}\right)-d^{2} z_{00}^{2} / 4=0 .
\end{aligned}
$$

In this way, $\mathcal{V}_{d}$ is seen as a quadric of $\mathbf{P}^{N}$ which will be called quadric of segments (QoS) of length $d$.

\section{Basic PRoperties of the QoS}

The QoS defined in (13) can be written in matrix form as

$$
\sigma(\mathbf{x}, \mathbf{y})^{\top} \mathbf{C}^{\text {euc }} \sigma(\mathbf{x}, \mathbf{y})=0
$$

for certain matrix

$$
\mathrm{C}^{\mathrm{euc}}=\mathrm{C}_{1}^{\mathrm{euc}}+\frac{d^{2}}{2} \mathrm{C}_{2}^{\mathrm{euc}}
$$

where

$$
\mathrm{C}_{1}^{\mathrm{euc}}=\left(\begin{array}{ccc}
\mathrm{A} & & \\
& \mathrm{I}_{n} & \\
& & 0
\end{array}\right),
$$

the $n+1$-order matrix $\mathrm{A}$ is given by

$$
A=\left(\begin{array}{cccc}
0 & -1 & \cdots & -1 \\
-1 & 0 & & 0 \\
\vdots & & & \\
-1 & 0 & & 0
\end{array}\right)
$$

and the only non-zero entry of $\mathrm{C}_{2}^{\text {euc }}$ is the upper left coordinate which takes the value -1 . Note that rank $\mathrm{C}^{\text {euc }}=\operatorname{rank} \mathrm{C}_{1}^{\text {euc }}=n+2$, while rank $\mathrm{C}_{2}^{\text {euc }}=1$.

A change of coordinates $\mathbf{x}^{\prime}=\mathrm{Hx}$ induces a change or coordinates in $\mathbf{P}^{N}$

$$
\mathbf{s}^{\prime}=\tilde{\mathrm{H}} \mathbf{s}
$$

where $\tilde{\mathrm{H}}$ is defined by the relations

$$
\sigma\left(\mathbf{x}^{\prime}, \mathbf{y}^{\prime}\right)=\tilde{\mathrm{H}} \sigma(\mathbf{x}, \mathbf{y}) .
$$

Denoting by $\mathbf{e}_{i}$ the elements of the canonical basis of $\mathbf{C}^{n+1}$ and by $\mathbf{E}_{k}$ those of the canonical basis of $\mathbf{C}^{N+1}$, we have $\sigma\left(\mathbf{e}_{i}, \mathbf{e}_{j}\right)=\sqrt{1+\delta_{i j}} \mathbf{E}_{k(i, j)}, \delta_{i j}$ being Kronecker's symbol. 
Substituting in (18) $\mathbf{x}=\mathbf{e}_{i}, \mathbf{y}=\mathbf{e}_{j}$ we obtain the explicit expression

$$
\tilde{\mathrm{H}}=\left(\frac{\sigma\left(\mathbf{h}_{0}, \mathbf{h}_{0}\right)}{\sqrt{2}}, \ldots, \frac{\sigma\left(\mathbf{h}_{n}, \mathbf{h}_{n}\right)}{\sqrt{2}}, \sigma\left(\mathbf{h}_{0}, \mathbf{h}_{1}\right), \sigma\left(\mathbf{h}_{0}, \mathbf{h}_{2}\right), \ldots, \sigma\left(\mathbf{h}_{n-1}, \mathbf{h}_{n}\right)\right)
$$

(cf. equation (4)).

The following property of $\tilde{\mathrm{H}}$ will be of importance in what follows:

Result 5.1. For any square matrix $\mathrm{H}$ we have that

$$
\widetilde{\mathrm{H}^{\top}}=\widetilde{\mathrm{H}}^{\top} \text {. }
$$

Proof. By definition of $\tilde{\mathrm{H}}$ we have

$$
\begin{aligned}
& \left\langle\sigma(\mathbf{u}, \mathbf{v}), \tilde{\mathrm{H}}^{\top} \sigma(\mathbf{w}, \mathbf{z})\right\rangle=\langle\tilde{\mathrm{H}} \sigma(\mathbf{u}, \mathbf{v}), \sigma(\mathbf{w}, \mathbf{z})\rangle=\langle\sigma(\mathrm{H} \mathbf{u}, \mathrm{H} \mathbf{v}), \sigma(\mathbf{w}, \mathbf{z})\rangle \\
= & \langle\mathrm{S}(\mathrm{H} \mathbf{u}, \mathrm{H} \mathbf{v}), \mathrm{S}(\mathbf{w}, \mathbf{z})\rangle=\langle\mathrm{H} \mathbf{u}, \mathbf{w}\rangle\langle\mathrm{H} \mathbf{v}, \mathbf{z}\rangle+\langle\mathrm{H} \mathbf{u}, \mathbf{z}\rangle\langle\mathrm{H} \mathbf{v}, \mathbf{w}\rangle \\
= & \left\langle\mathbf{u}, \mathrm{H}^{\top} \mathbf{w}\right\rangle\left\langle\mathbf{v}, \mathrm{H}^{\top} \mathbf{z}\right\rangle+\left\langle\mathbf{u}, \mathrm{H}^{\top} \mathbf{z}\right\rangle\left\langle\mathbf{v}, \mathrm{H}^{\top} \mathbf{w}\right\rangle=\left\langle\mathrm{S}(\mathbf{u}, \mathbf{v}), \mathrm{S}\left(\mathrm{H}^{\top} \mathbf{w}, \mathrm{H}^{\top} \mathbf{z}\right)\right\rangle \\
= & \left\langle\sigma(\mathbf{u}, \mathbf{v}), \sigma\left(\mathrm{H}^{\top} \mathbf{w}, \mathrm{H}^{\top} \mathbf{z}\right)\right\rangle=\left\langle\sigma(\mathbf{u}, \mathbf{v}), \widetilde{\mathrm{H}^{\top}} \sigma(\mathbf{w}, \mathbf{z})\right\rangle .
\end{aligned}
$$

Since the image of $\sigma$ spans the whole space, we have the desired result.

If $\mathbf{x}^{\text {euc }}=\mathrm{H} \mathbf{x}$ is the change of coordinates between Euclidean and arbitrary projective coordinates, the matrix of the QoS in the latter coordinate system is

$$
\mathrm{C}=\tilde{\mathrm{H}}^{\top} \mathrm{C}^{\mathrm{euc}} \tilde{\mathrm{H}}
$$

and

$$
\mathrm{C}_{i}=\tilde{\mathrm{H}}^{\top} \mathrm{C}_{i}^{\mathrm{euc}} \tilde{\mathrm{H}} .
$$

Result 5.2 (Geometric interpretation of $\mathrm{C}_{1}$ and $\mathrm{C}_{2}$ ). (1) $\sigma(\mathbf{x}, \mathbf{y})^{\top} \mathbf{C}_{1} \sigma(\mathbf{x}, \mathbf{y})=0$ if and only if the line $\mathbf{x y}$ intersects the absolute quadric $\Omega_{\infty}$.

(2) $\sigma(\mathbf{x}, \mathbf{y})^{\top} C_{2} \sigma(\mathbf{x}, \mathbf{y})=0$ if and only if either $\mathbf{x}$ or $\mathbf{y}$ lie on the hyperplane at infinity $\pi_{\infty}$

Proof. To prove the first statement we use Euclidean coordinates and observe that the intersection of a line xy with the hyperplane at infinity (and not contained in it) is the point $y_{0} \mathbf{x}-x_{0} \mathbf{y}$, which lies in the absolute quadric $\Omega_{\infty}$ if and only if

$$
0=\sum_{i=1}^{n}\left(x_{i} y_{0}-y_{i} x_{0}\right)^{2}=\sigma(\mathbf{x}, \mathbf{y})^{\top} \mathbf{C}_{1} \sigma(\mathbf{x}, \mathbf{y})
$$

The second statement is easily proved in Euclidean coordinates, since

$$
\sigma(\mathbf{x}, \mathbf{y})^{\top} \mathbf{C}_{2} \sigma(\mathbf{x}, \mathbf{y})=-x_{0}^{2} y_{0}^{2} .
$$

We are now in conditions to show how $\mathrm{C}_{2}$ is related with the plane at infinity:

Result 5.3. Let $\boldsymbol{\pi}_{\infty}$ be the coordinates of the plane at infinity. Then,

$$
\mathrm{C}_{2} \sim \sigma\left(\boldsymbol{\pi}_{\infty}, \boldsymbol{\pi}_{\infty}\right) \sigma\left(\boldsymbol{\pi}_{\infty}, \boldsymbol{\pi}_{\infty}\right)^{\top}
$$


Proof. Let us denote by $\mathbf{k}_{i}, i=0,1,2,3$, the rows of $\mathrm{H}$. Since

$$
\mathrm{C}_{2}=\tilde{\mathrm{H}}^{\top} \mathrm{C}_{2}^{\mathrm{euc}} \tilde{\mathrm{H}}
$$

using (19) and the expression of $\mathrm{C}_{2}^{\text {euc }}$ we have

$$
\mathrm{C}_{2}=-\left(\sigma\left(\mathbf{k}_{0}, \mathbf{k}_{0}\right) / \sqrt{2}\right)\left(\sigma\left(\mathbf{k}_{0}, \mathbf{k}_{0}\right) / \sqrt{2}\right)^{\top}=-\frac{1}{2} \sigma\left(\mathbf{k}_{0}, \mathbf{k}_{0}\right) \sigma\left(\mathbf{k}_{0}, \mathbf{k}_{0}\right)^{\top} .
$$

Being $\mathbf{k}_{0} \sim \boldsymbol{\pi}_{\infty}$, the result follows.

Remark 5.1. Using result 5.3 we see that the expression

$$
\mathrm{C}_{2}(\mathbf{x})=\sigma(\mathbf{x}, \mathbf{x}) \sigma(\mathbf{x}, \mathbf{x})^{\top}
$$

parametrizes all the possible matrices $\mathrm{C}_{2}$ as we change the projective coordinate system.

It seems natural to ask if the knowledge of the zeros of the QoS along the variety of segments

$$
\operatorname{im} \sigma=\left\{\sigma(\mathbf{x}, \mathbf{y}): \mathbf{x}, \mathbf{y} \in \mathbf{P}^{n}\right\} \subset \mathbf{P}^{N},
$$

determines the QoS completely, for it may exist a different quadric in $\mathbf{P}^{N}$ intersecting $\operatorname{im} \sigma$ in the same locus. In this section we will see that this is not the case, which is relevant for us since it will allow to obtain the QoS just from observations of segments of length $d$.

Result 5.4. Let $\mathrm{C}$ and $\mathrm{C}^{\prime}$ be two quadrics of $\mathbf{P}^{N}$ having the same zeros along $\operatorname{im} \sigma$, i.e.,

$$
\sigma(\mathbf{x}, \mathbf{y})^{\top} \mathbf{C} \sigma(\mathbf{x}, \mathbf{y})=0 \Longleftrightarrow \sigma(\mathbf{x}, \mathbf{y})^{\top} \mathbf{C}^{\prime} \sigma(\mathbf{x}, \mathbf{y})=0 \text {. }
$$

Then $\mathbf{C}=\lambda \cdot \mathrm{C}^{\prime}$ for some $\lambda \in \mathbf{C} \backslash\{0\}$.

The proof of this result is given in the appendix.

\section{LINEAR SPACES SPANNED BY THE MATRICES $\mathrm{C}_{1}$ AND $\mathrm{C}_{2}$}

With the aim of obtaining linear parametrizations of the matrices $\mathrm{C}_{1}$ and $\mathrm{C}_{2}$, in this section we study the linear spaces they span. The following result will be one of the keys for the linear computation of the QoS that we address in section 9.

Result 6.1. The space of symmetric matrices of order $N+1$ can be decomposed as

$$
\operatorname{Sym}(N+1)=S_{1} \oplus S_{2}
$$

where each $S_{i}$ is defined as the vector subspace of $\operatorname{Sym}(N+1)$ generated by all the possible matrices $\mathrm{C}_{i}$ obtained from $\mathrm{C}_{i}^{\text {euc }}$ using admissible changes of coordinates (22). Moreover, $S_{1}$ and $S_{2}$ are orthogonal with respect the natural metric defined in (2) and their dimensions are

$$
\begin{aligned}
\operatorname{dim} S_{1} & =\frac{1}{12} n(n+1)^{2}(n+2), \\
\operatorname{dim} S_{2} & =\left(\begin{array}{c}
n+4 \\
4
\end{array}\right) .
\end{aligned}
$$

Finally, for a given length $d$ the linear span of all the possible matrices $\mathrm{C}$ is the total space $\operatorname{Sym}(N+1)$. 
Proof. Using result 5.3 we can parametrize the possible $\mathrm{C}_{2}$ matrices as

$$
\mathrm{C}_{2}(\mathbf{x}) \sim \sigma(\mathbf{x}, \mathbf{x}) \sigma(\mathbf{x}, \mathbf{x})^{\top}
$$

It readily follows from the definition of $\sigma(\mathbf{x}, \mathbf{y})$ that each entry of $\sigma(\mathbf{x}, \mathbf{x}) \sigma(\mathbf{x}, \mathbf{x})^{\top}$ is proportional to a degree-four monomial in the variables $x_{0}, \ldots, x_{n}$ and that none of such monomials is missed, so $S_{2}$ has the same dimension as the vector space of degree-four homogeneous polynomials, i.e., $\left(\begin{array}{c}n+4 \\ 4\end{array}\right)$.

Let us see now that $S_{1}$ is orthogonal to $S_{2}$. Since the distance of a point to itself is zero, the equation

$$
\sigma(\mathbf{x}, \mathbf{x})^{\top}\left(\mathbf{C}_{1}+0 \mathbf{C}_{2}\right) \sigma(\mathbf{x}, \mathbf{x})=\sigma(\mathbf{x}, \mathbf{x})^{\top} \mathbf{C}_{1} \sigma(\mathbf{x}, \mathbf{x})=0
$$

holds true for all $\mathbf{x}$. This provides a set of linear equations satisfied by the matrices $\mathrm{C}_{1}$ and therefore by any element of $S_{1}$. Using (23) and the product (2) to write equation (27) as

$$
0=\operatorname{trace}\left(\sigma(\mathbf{x}, \mathbf{x})^{\top} \mathrm{C}_{1} \sigma(\mathbf{x}, \mathbf{x})\right)=\operatorname{trace}\left(\sigma(\mathbf{x}, \mathbf{x}) \sigma(\mathbf{x}, \mathbf{x})^{\top} \mathrm{C}_{1}\right) \sim\left\langle\mathrm{C}_{2}(\mathbf{x}), \mathbf{C}_{1}\right\rangle,
$$

we conclude that $S_{1} \perp S_{2}$. From this we obtain an upper bound for $\operatorname{dim} S_{1}$ :

$\operatorname{dim} S_{1} \leq \operatorname{dim} \operatorname{Sym}(N+1)-\operatorname{dim} S_{2}=\frac{(N+1)(N+2)}{2}-\left(\begin{array}{c}n+4 \\ 4\end{array}\right)=\frac{1}{12} n(n+1)^{2}(n+2)$.

Let us see that, in fact, $\operatorname{dim} S_{1}$ equals this upper-bound. Using (19) and (16) we can write $\mathrm{C}_{1}$ as follows:

$$
\begin{aligned}
\mathrm{C}_{1}= & \tilde{\mathrm{H}}^{\top} \mathrm{C}_{1}^{\text {euc }} \tilde{\mathrm{H}}=\widetilde{\mathrm{H}^{\top}} \mathrm{C}_{1}^{\text {euc }} \widetilde{\mathrm{H}^{\top}}= \\
& \sum_{i=1}^{n}\left(\sigma\left(\mathbf{k}_{0}, \mathbf{k}_{i}\right) \sigma\left(\mathbf{k}_{0}, \mathbf{k}_{i}\right)^{\top}-\sigma\left(\mathbf{k}_{0}, \mathbf{k}_{0}\right) \sigma\left(\mathbf{k}_{i}, \mathbf{k}_{i}\right)^{\top}-\sigma\left(\mathbf{k}_{i}, \mathbf{k}_{i}\right) \sigma\left(\mathbf{k}_{0}, \mathbf{k}_{0}\right)^{\top}\right),
\end{aligned}
$$

where $\mathbf{k}_{i}$ denote the rows of $\mathrm{H}=\left(h_{i j}\right)$. Let us see that the monomials involving only $\mathbf{k}_{0}$ and $\mathbf{k}_{1}$ are enough to attain the required dimension of $S_{2}$. We denote

$$
\mathrm{E}^{i}=\sigma\left(\mathbf{k}_{0}, \mathbf{k}_{i}\right) \sigma\left(\mathbf{k}_{0}, \mathbf{k}_{i}\right)^{\top}-\sigma\left(\mathbf{k}_{0}, \mathbf{k}_{0}\right) \sigma\left(\mathbf{k}_{i}, \mathbf{k}_{i}\right)^{\top}-\sigma\left(\mathbf{k}_{i}, \mathbf{k}_{i}\right) \sigma\left(\mathbf{k}_{0}, \mathbf{k}_{0}\right)^{\top}
$$

So

$$
\mathrm{C}_{1}=\sum_{i=1}^{n} \mathrm{E}^{i} .
$$

Observe that since the topological closure of $\mathrm{GL}(n+1)$ is the set $\mathbf{M}_{n+1}$ of all square matrices of order $n+1$, we have

$$
\operatorname{dim} \operatorname{Span}\left\{\mathrm{C}_{1}(\mathrm{H}): \mathrm{H} \in \mathrm{GL}(n+1)\right\}=\operatorname{dim} \operatorname{Span}\left\{\mathrm{C}_{1}(\mathrm{H}): \mathrm{H} \in \mathbf{M}_{n+1}\right\} .
$$

Since

$$
\left\{\mathrm{C}_{1}(\mathrm{H}): \mathrm{H} \in \mathbf{M}_{n+1}\right\} \supset\left\{\mathrm{E}^{1}(\mathrm{H}): \mathrm{H} \in \mathbf{M}_{n+1}\right\},
$$

we have that

$$
\operatorname{dim} \operatorname{Span}\left\{\mathrm{C}_{1}(\mathrm{H}): \mathrm{H} \in \mathbf{M}_{n+1}\right\} \geq \operatorname{dim} \operatorname{Span}\left\{\mathrm{E}^{1}(\mathrm{H}): \mathrm{H} \in \mathbf{M}_{n+1}\right\} \geq \frac{1}{12} n(n+1)^{2}(n+2),
$$

where the last inequality is the content of lemma A.3.

Finally, the proof that

$$
\operatorname{Span}\{\mathrm{C}(\mathrm{H}): \mathrm{H} \in \mathrm{GL}(n+1)\}=\operatorname{Sym}(N+1)
$$


is entirely similar to the result we have just proved for $\mathrm{C}_{1}$ using that

$$
\mathrm{C}=-\frac{d^{2}}{4} \mathrm{E}^{0}+\sum_{i=1}^{n} \mathrm{E}^{i} .
$$

where

and checking that the $\left(\begin{array}{c}n+4 \\ 4\end{array}\right)$ derivatives

$$
\mathrm{E}^{0}=\sigma\left(\mathbf{k}_{0}, \mathbf{k}_{0}\right) \sigma\left(\mathbf{k}_{0}, \mathbf{k}_{0}\right)^{\top}
$$

$$
\mathrm{E}_{i j k l}^{0}=\frac{\partial^{4} \mathrm{E}^{0}}{\partial h_{i 0} \partial h_{j 0} \partial h_{k 0} \partial h_{l 0}}
$$

are linearly independent of the derivatives (48).

7. Extracting Affine and Euclidean Structure from the Quadric of SEGMENTS

The QoS is determined by the pair of matrices $\left(\mathrm{C}_{1}, \mathrm{C}_{2}\right)$ defined up to a common scale factor. Changing the pair $\left(\mathrm{C}_{1}, \mathrm{C}_{2}\right)$ by $\left(\mathrm{C}_{1}, \lambda \mathrm{C}_{2}\right)$ amounts to changing the unit of length, since from $(14,15)$

$$
d^{2}=-2 \frac{\sigma(\mathbf{x}, \mathbf{y})^{\top} \mathbf{C}_{1} \sigma(\mathbf{x}, \mathbf{y})}{\sigma(\mathbf{x}, \mathbf{y})^{\top} \mathbf{C}_{2} \sigma(\mathbf{x}, \mathbf{y})}
$$

Therefore, the QoS determines not only the similarity group, as the absolute quadric does, but also the group of isometries of space. The following results describes the relationship between the QoS and the usual stratified geometry of space.

Result 7.1. $A(n+1) \times(n+1)$ regular matrix $\mathrm{H}$ represents

(1) a similarity if and only if $\tilde{\mathrm{H}}^{\top} \mathrm{C}_{1} \tilde{\mathrm{H}} \sim \mathrm{C}_{1}$.

(2) an affinity if and only if $\tilde{\mathrm{H}}^{\top} \mathrm{C}_{2} \tilde{\mathrm{H}} \sim \mathrm{C}_{2}$.

(3) an isometry if and only if $\left(\tilde{\mathrm{H}}^{\top} \mathrm{C}_{1} \tilde{\mathrm{H}}, \tilde{\mathrm{H}}^{\top} \mathrm{C}_{2} \tilde{\mathrm{H}}\right) \sim\left(\mathrm{C}_{1}, \mathrm{C}_{2}\right)$ or, equivalently, if and only if $\tilde{\mathrm{H}}^{\top} \mathrm{C} \tilde{\mathrm{H}} \sim \mathrm{C}$.

Proof. The fact that $\tilde{\mathrm{H}}$ leaves invariant $\mathrm{C}_{1}\left(\right.$ resp. $\left.\mathrm{C}_{2}, \mathrm{C}\right)$ is, due to result 5.4 , equivalent to the fact that the set of segments $\{\mathbf{x}, \mathbf{y}\} \operatorname{such}$ that $\operatorname{dist}(\mathbf{x}, \mathbf{y})=0(\operatorname{resp} \cdot \operatorname{dist}(\mathbf{x}, \mathbf{y})=\infty$, $\operatorname{dist}(\mathbf{x}, \mathbf{y})=d)$, is $\mathrm{H}$-invariant. In the first case, it is equivalent to the H-invariance of the set of cones through the absolute quadric at infinity $\Omega_{\infty}$, which in turn is equivalent to the invariance of $\Omega_{\infty}$ itself.

The H-invariance of $\operatorname{dist}(\mathbf{x}, \mathbf{y})=\infty$ is equivalent to the H-invariance of the hyperplane at infinity, i.e., to $\mathrm{H}$ being an affinity. Finally, the invariance of segments of length $d$ characterizes isometries, which is equivalent to $\left(\tilde{\mathrm{H}}^{\top} \mathrm{C}_{1} \tilde{\mathrm{H}}, \tilde{\mathrm{H}}^{\top} \mathrm{C}_{2} \tilde{\mathrm{H}}\right) \sim\left(\mathrm{C}_{1}, \mathrm{C}_{2}\right)$.

Now let us see several techniques to extract affine and Euclidean geometry from the QoS. First let us write

$$
\sigma(\mathbf{x}, \mathbf{y})^{\top} \mathbf{C} \sigma(\mathbf{x}, \mathbf{y})=\mathbf{x}^{\top} \mathbf{A}_{\mathbf{y}} \mathbf{x}
$$

$\mathrm{A}_{\mathbf{y}}$ is the matrix of the radius $d$ sphere centered at $\mathbf{y}$, being its entries degree-two polynomials in the coordinates of $\mathbf{y}$ :

$$
\left(\mathrm{A}_{\mathbf{y}}\right)_{i j}=\sum_{k, l=0}^{n} \mathrm{C}_{i k}^{j l} y_{k} y_{l}
$$

where the coefficients $\mathrm{C}_{i k}^{j l}$ were defined by $(47)$. 
The plane at infinity can be recovered as the polar hyperplane of the center of the sphere, i.e.,

$$
\pi_{\infty}=\mathrm{A}_{\mathbf{y}} \mathbf{y} .
$$

The Euclidean geometry can be recovered as well, for example obtaining the dual absolute quadric (DAQ). As is well known, the DAQ can be defined as the mapping assigning to each hyperplane $\boldsymbol{\pi}$ its orthogonal point at infinity $\mathbf{Q}_{\infty}^{*} \boldsymbol{\pi}$. Taking into account that the orthogonal point at infinity of $\boldsymbol{\pi}$ can be obtained as the intersection of $\boldsymbol{\pi}_{\infty}$ with the line defined by $\mathbf{y}$ and the pole of the the hyperplane $\boldsymbol{\pi}$ with respect to the sphere, it is not hard to arrive to the following expression for the DAQ:

$$
\mathbf{Q}_{\infty}^{*}=\left(\mathbf{y}^{\top} \mathrm{A}_{\mathbf{y}} \mathbf{y}\right) \mathrm{A}_{\mathbf{y}}^{*}-\operatorname{det}\left(\mathrm{A}_{\mathbf{y}}\right) \mathbf{y} \mathbf{y}^{\top} \text {. }
$$

Both (31) and (32) are not completely satisfactory because of their dependency on $\mathbf{y}$; in fact (31) has degree 3 and (32) has degree $2(n+2)$. In this section we provide alternative solutions without this drawback.

7.1. Extracting the plane at infinity from $\mathrm{C}_{2}$. Closed form expressions of the plane at infinity from the coefficients of $\mathrm{C}_{2}$ are given by the following result.

Result 7.2. The plane at infinity can be obtained from $\mathrm{C}_{2}$ as follows: Take any $\alpha, \beta, \gamma \in$ $\{0, \ldots, n\}$, then

$$
\pi_{\infty} \sim\left(\begin{array}{c}
\sigma\left(\mathbf{e}_{0}, \mathbf{e}_{\alpha}\right)^{\top} \mathrm{C}_{2} \sigma\left(\mathbf{e}_{\beta}, \mathbf{e}_{\gamma}\right) \\
\vdots \\
\sigma\left(\mathbf{e}_{n}, \mathbf{e}_{\alpha}\right)^{\top} \mathrm{C}_{2} \sigma\left(\mathbf{e}_{\beta}, \mathbf{e}_{\gamma}\right)
\end{array}\right)
$$

Proof. Let $\mathbf{x}$ be the coordinates of the plane at infinity. Using again result 5.3 we have that

$$
\mathrm{C}_{2}(\mathbf{x})=\sigma(\mathbf{x}, \mathbf{x}) \sigma(\mathbf{x}, \mathbf{x})^{\top}
$$

Then

$$
\sigma\left(\mathbf{e}_{i}, \mathbf{e}_{\alpha}\right)^{\top} \mathbf{C}_{2} \sigma\left(\mathbf{e}_{\beta}, \mathbf{e}_{\gamma}\right)=\sigma\left(\mathbf{e}_{i}, \mathbf{e}_{\alpha}\right)^{\top} \sigma(\mathbf{x}, \mathbf{x}) \sigma(\mathbf{x}, \mathbf{x})^{\top} \sigma\left(\mathbf{e}_{\beta}, \mathbf{e}_{\gamma}\right),
$$

then, using $(2,5,6)$

$$
\begin{aligned}
\sigma\left(\mathbf{e}_{i}, \mathbf{e}_{\alpha}\right)^{\top} \sigma(\mathbf{x}, \mathbf{x}) & =\left\langle\sigma\left(\mathbf{e}_{i}, \mathbf{e}_{\alpha}\right), \sigma(\mathbf{x}, \mathbf{x})\right\rangle \\
& =\left\langle\mathbf{S}\left(\mathbf{e}_{i}, \mathbf{e}_{\alpha}\right), \mathbf{S}(\mathbf{x}, \mathbf{x})\right\rangle \\
& =\frac{1}{2} \operatorname{trace}\left(\left(\mathbf{e}_{i} \mathbf{e}_{\alpha}^{\top}+\mathbf{e}_{\alpha} \mathbf{e}_{i}^{\top}\right)\left(2 \mathbf{x} \mathbf{x}^{\top}\right)\right) \\
& =2 x_{i} x_{\alpha}
\end{aligned}
$$

therefore

$$
\sigma\left(\mathbf{e}_{i}, \mathbf{e}_{\alpha}\right)^{\top} \mathrm{C}_{2} \sigma\left(\mathbf{e}_{\beta}, \mathbf{e}_{\gamma}\right)=4 x_{i} x_{\alpha} x_{\beta} x_{\gamma}
$$

and the result follows.

7.2. Extracting the DAQ from $\mathrm{C}_{1}$. Since $\mathrm{C}_{1}$ gives all the cones with base the absolute quadric at infinity, it should be possible to recover the DAQ from it. Next result and the subsequent discussion shows that this task can be accomplished in a very clean algebraic way. 
Result 7.3. Let us suppose that the coordinate point $\mathbf{e}_{\alpha}$ does not lie in $\boldsymbol{\pi}_{\infty}$. Then the planes of the dual absolute quadric $\mathbf{Q}_{\infty}^{*}$ passing through the coordinate point $\mathbf{e}_{\alpha}$ are defined by the matrix

$$
\mathbf{Q}_{\alpha}^{*}=\left(\left(\sigma\left(\mathbf{e}_{i}, \mathbf{e}_{\alpha}\right)^{\top} \mathbf{C}_{1} \sigma\left(\mathbf{e}_{j}, \mathbf{e}_{\alpha}\right)\right)_{\substack{0 \leq i, j \leq n \\ i, j \neq \alpha}}\right)^{*}
$$

in the following sense: a hyperplane $\boldsymbol{\pi}$ belongs to $\mathbf{Q}_{\infty}^{*} \cap\left\{\pi_{\alpha}=0\right\}$ if and only if

$$
\pi_{\alpha}=0, \text { and } \boldsymbol{\pi}_{\alpha}^{\top} \mathbf{Q}_{\alpha}^{*} \boldsymbol{\pi}_{\alpha}=0
$$

where $\boldsymbol{\pi}_{\alpha}=\left(\pi_{0}, \ldots, \pi_{\alpha-1}, \pi_{\alpha+1}, \ldots, \pi_{n}\right)^{\top}$.

Proof. The equation of the cone $V_{\mathbf{y}}$ of vertex $\mathbf{y}$ passing through the absolute conic is $\sigma(\mathbf{x}, \mathbf{y})^{\top} \mathbf{C}_{1} \sigma(\mathbf{x}, \mathbf{y})=0$. Therefore the intersection $V_{\mathbf{e}_{\alpha}} \cap\left\{x_{\alpha}=0\right\}$ is given by the equations

$$
\begin{aligned}
x_{\alpha} & =0, \\
\sigma\left(\mathbf{x}, \mathbf{e}_{\alpha}\right)^{\top} \mathrm{C}_{1} \sigma\left(\mathbf{x}, \mathbf{e}_{\alpha}\right) & =\sum_{\substack{i, j=0 \\
i, j \neq \alpha}}^{n} x_{i} x_{j} \sigma\left(\mathbf{e}_{i}, \mathbf{e}_{\alpha}\right)^{\top} \mathrm{C}_{1} \sigma\left(\mathbf{e}_{j}, \mathbf{e}_{\alpha}\right)=0
\end{aligned}
$$

Using $\left(x_{0}, \ldots, x_{\alpha-1}, x_{\alpha+1}, \ldots, x_{n}\right)^{\top}$ as coordinates in the hyperplane $x_{\alpha}=0$ we see that the matrix

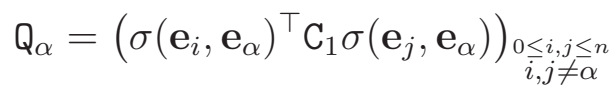

defines the quadric $V_{\mathbf{e}_{\alpha}} \cap\left\{x_{\alpha}=0\right\}$ which, since we assume $\mathbf{e}_{\alpha} \notin \boldsymbol{\pi}_{\infty}$, is non-degenerated. Hence its adjoint matrix $\mathrm{Q}_{\alpha}^{*}$ determine its tangent spaces $\boldsymbol{\pi}_{\alpha}=\left(\pi_{0}, \ldots, \pi_{\alpha-1}, \pi_{\alpha+1}, \ldots, \pi_{n}\right)^{\top}$. The hyperplanes defined by these tangent spaces and $\mathbf{e}_{\alpha}$ are the hyperplanes of $\mathbf{Q}_{\infty}^{*}$ passing through $\mathbf{e}_{\alpha}$, i.e., those $\boldsymbol{\pi}=\left(\pi_{0}, \ldots, \pi_{\alpha-1}, 0, \pi_{\alpha+1}, \ldots, \pi_{n}\right)^{\top}$ such that

$$
\boldsymbol{\pi}_{\alpha}^{\top} \mathrm{Q}_{\alpha}^{*} \boldsymbol{\pi}_{\alpha}=0 .
$$

Let $M$ be a matrix and let us denote by $\Lambda_{\alpha}(M)$ the submatrix resulting of suppressing its $\alpha$ row and column. As a consequence of the last result, we see that if none of the $\mathbf{e}_{\alpha} \notin \boldsymbol{\pi}_{\infty}$ then there exists non-zero constants $\lambda_{\alpha}$ such that

$$
\Lambda_{\alpha}\left(\mathrm{Q}_{\infty}^{*}\right)=\lambda_{\alpha} \mathrm{Q}_{\alpha}^{*}, \alpha=0, \ldots, n .
$$

From these overdetermined system of linear equations it is immediate to recover the DAQ from $\mathrm{C}_{1}$. Note that if any of the $\mathbf{e}_{\alpha} \in \boldsymbol{\pi}_{\infty}$, which can be detected by the lower rank of $\mathrm{Q}_{\alpha}$, a projective change of coordinates would carry everything to a generic non-singular position.

7.3. Kernels of $\mathrm{C}, \mathrm{C}_{1}$ and $\mathrm{C}_{2}$. The kernel of a quadric is a projective invariant of the quadric which is easy to compute. Next result provides a geometrical interpretation of the kernels of $\mathrm{C}, \mathrm{C}_{1}$ and $\mathrm{C}_{2}$.

Result 7.4. The segments of the kernel of the quadric $\mathrm{C}_{1}$ are those whose ends are points at infinity corresponding to orthogonal directions, while the segments of the kernel of $\mathrm{C}_{2}$ are those such that at least one of their ends lie on the plane at infinity. Finally, the kernel of $\mathrm{C}$ coincide with that of $\mathrm{C}_{1}$. 
Proof. Using Euclidean coordinates, it is immediate to check that $\mathrm{C}_{1}^{\text {euc }} \sigma\left(\mathbf{x}^{\text {euc }}, \mathbf{y}^{\text {euc }}\right)=0$ is equivalent to the set of equations

$$
\begin{aligned}
\sum_{i=1}^{n} x_{i}^{\mathrm{euc}} y_{i}^{\mathrm{euc}} & =0, \\
x_{0}^{\mathrm{euc}} y_{0}^{\mathrm{euc}} & =0, \\
x_{0}^{\mathrm{euc}} y_{j}^{\mathrm{euc}}+y_{0}^{\mathrm{euc}} x_{j}^{\text {euc }} & =0, j=1, \ldots, n,
\end{aligned}
$$

from which the first statement readily follows. The proof of the second is similar, since $\mathrm{C}_{2}^{\text {euc }} \sigma\left(\mathbf{x}^{\mathrm{euc}}, \mathbf{y}^{\mathrm{euc}}\right)=0$ if and only if $x_{0} y_{0}=0$. Finally, it is immediate to check that the kernel of $\mathrm{C}$ has identical equations as that of $\mathrm{C}_{1}$.

Note that a particular consequence of the last result is that the equations

$$
\mathrm{C}_{1} \sigma(\mathbf{x}, \mathbf{x})=0
$$

are those of the absolute quadric at infinity.

\section{The Quadric of Circles and the Absolute Quadratic Complex}

We recall that the Plücker coordinates $[4$, p. 70$]$ of the line defined by $\mathbf{x}$ and $\mathbf{y}$ are the numbers $p_{i j}$ given by

$$
p_{i j}=x_{i} y_{j}-x_{j} y_{i}
$$

The identity

$$
p_{i j} p_{k l}=z_{i l} z_{j k}-z_{i k} z_{j l}
$$

shows that any quadric in Plücker coordinates can be written as a quadric in terms of the symmetric coordinates $z_{i j}$, i.e., a quadric in the space of segments. A quadric in Plücker coordinates relevant for Euclidean reconstruction is the Absolute Quadratic Complex (AQC) $[12,17]$, given by the lines intersecting the absolute quadric at infinity. Next result shows that the $\mathrm{AQC}$ can be written in terms of $\mathrm{C}_{1}$ in a particularly satisfactory way. Note that since the AQC theory has been developed only for dimension 3 some of the results will be restricted to this particular case.

Result 8.1. The quadric $\mathrm{C}_{1}$ coincides with the absolute quadratic complex $\Sigma$ in the following sense, given any vectors $\mathbf{x}, \mathbf{y}$ we have

$$
(\mathbf{x} \wedge \mathbf{y})^{\top} \Sigma(\mathbf{x} \wedge \mathbf{y})=\sigma(\mathbf{x}, \mathbf{y})^{\top} \mathbf{C}_{1} \sigma(\mathbf{x}, \mathbf{y})
$$

Besides, the coefficients of $\mathrm{C}_{1}$ can be obtained from the coefficients of $\Sigma$ according to the equation

$$
\mathrm{C}_{i j}^{k l}=\Sigma_{i k}^{l j}+\Sigma_{i l}^{k j}
$$

where $\mathrm{C}_{i j}^{k l}$ is defined by equation (47) and, analogously,

$$
\Sigma_{i j}^{k l}=\left(\mathbf{e}_{i} \wedge \mathbf{e}_{j}\right)^{\top} \Sigma\left(\mathbf{e}_{k} \wedge \mathbf{e}_{l}\right) .
$$

Conversely, assuming $n=3$, the AQC can be recovered from the $Q O S$ as follows

$$
\Sigma_{i j}^{k l}=\frac{1}{3}\left(\mathrm{C}_{i l}^{j k}-\mathrm{C}_{i k}^{j l}\right) .
$$


Proof. Since the identity we want to prove is geometric, i.e., invariant under linear coordinate changes, it is enough to check it for an Euclidean coordinate system:

$$
\begin{aligned}
\sigma\left(\mathbf{x}^{\mathrm{euc}}, \mathbf{y}^{\mathrm{euc}}\right)^{\top} \mathrm{C}_{1}^{\mathrm{euc}} \sigma\left(\mathbf{x}^{\mathrm{euc}}, \mathbf{y}^{\mathrm{euc}}\right) & =\sum_{i=1}^{n}\left(x_{i}^{\mathrm{euc}} y_{0}^{\mathrm{euc}}-y_{i}^{\mathrm{euc}} x_{0}^{\mathrm{euc}}\right)^{2} \\
& =\sum_{i=1}^{n} p_{i 0}^{2} \\
& =\left(\mathbf{x}^{\mathrm{euc}} \wedge \mathbf{y}^{\mathrm{euc}}\right)^{\top} \Sigma^{\mathrm{euc}}\left(\mathbf{x}^{\mathrm{euc}} \wedge \mathbf{y}^{\mathrm{euc}}\right)
\end{aligned}
$$

Equation (34) can be proved computing the derivative $\frac{\partial^{4}}{\partial x_{i} \partial y_{j} \partial x_{k} \partial y_{l}}$ of both sides of (33), which leads to the relation

$$
\mathrm{C}_{i j}^{k l}+\mathrm{C}_{i l}^{k j}=\Sigma_{i j}^{k l}+\Sigma_{i l}^{k j}
$$

Cyclical permutations of the indices $j, k, l$ in this equation results in

$$
\begin{aligned}
C_{i k}^{l j}+C_{i j}^{l k} & =\Sigma_{i k}^{l j}+\Sigma_{i j}^{l k}, \\
C_{i l}^{j k}+C_{i k}^{j l} & =\Sigma_{i l}^{j k}+\Sigma_{i k}^{j l},
\end{aligned}
$$

and calculating $(36)-(37)+(38)$, taking into account the symmetries of the $C_{i j}^{k l}$ and $\sum_{i j}^{k l}$, identity (34) is obtained.

In order to prove identity (35), we first recall that the AQC satisfies the additional linear constraint given by [24, Theorem 5.3]. It is not hard to see that this constraint is equivalent to

$$
\Sigma_{i j}^{k l}+\Sigma_{i l}^{j k}+\Sigma_{i k}^{l j}=0
$$

for any $i, j, k, l=0, \ldots, 3$. Now we define the linear space $\Sigma$ given by all tensors $\left(\Sigma_{i j}^{k l}\right)$ satisfying (39) together with symmetries

$$
\Sigma_{i j}^{k l}=-\Sigma_{j i}^{k l}=-\Sigma_{i j}^{l k}=\Sigma_{k l}^{i j} .
$$

Note that these tensors constitute a vector space of dimension $21-1=20$, which coincides with the dimension of $S_{1}$ given by result (6.1). So we have to check that (34) defines a linear isomorphism $\phi: \Sigma \rightarrow S_{1}$. First we prove that $\phi$ is well-defined, i.e., $\mathrm{C}=\phi(\Sigma) \in S_{1}$ for any $\Sigma \in \Sigma$. Using result (6.1) it is enough to see that the $\mathrm{C}$ defined by (34) satisfies $\left\langle\sigma(\mathbf{x}, \mathbf{x})^{\top} \sigma(\mathbf{x}, \mathbf{x}), \mathbf{C}\right\rangle=0$ for any $\mathbf{x}$. But we have

$$
\begin{aligned}
\left\langle\sigma(\mathbf{x}, \mathbf{x})^{\top} \sigma(\mathbf{x}, \mathbf{x}), \mathbf{C}\right\rangle & =\sigma(\mathbf{x}, \mathbf{x})^{\top} \mathrm{C} \sigma(\mathbf{x}, \mathbf{x})= \\
\sum_{i, j, k, l=0}^{3} x_{i} x_{j} x_{k} x_{l} \mathrm{C}_{i j}^{k l} & =\sum_{i, j, k, l=0}^{3} x_{i} x_{j} x_{k} x_{l}\left(\Sigma_{i k}^{l j}+\Sigma_{i l}^{k j}\right)=2(\mathbf{x} \wedge \mathbf{x}) \Sigma(\mathbf{x} \wedge \mathbf{x})=0
\end{aligned}
$$

and so $\phi$ is well-defined.

Now let us define the inverse of $\phi, \varphi: S_{1} \rightarrow \Sigma$, using relation (35). In order to prove that $\varphi$ is well-defined, we first note that relation (39) enforces $\mathrm{C}$ to satisfy the equation

$$
\mathrm{C}_{i j}^{k l}+\mathrm{C}_{i l}^{j k}+\mathrm{C}_{i k}^{l j}=0
$$

(which is also a consequence of (41)). Using (42) and the previously mentioned symmetries, it is immediate to check that $\varphi$ is actually well-defined, i.e., $\Sigma=\varphi(\mathrm{C}) \in \Sigma$. Finally 
let us prove that $\phi(\varphi(\mathrm{C}))=$ C. In fact, since $\mathrm{C}_{i j}^{k l}=\Sigma_{i k}^{l j}+\Sigma_{i l}^{k j}$ we have that

$$
\Sigma_{i k}^{l j}+\Sigma_{i l}^{k j}=\frac{1}{3}\left(\mathrm{C}_{i j}^{k l}-\mathrm{C}_{i l}^{k j}+\mathrm{C}_{i j}^{l k}-\mathrm{C}_{i k}^{l j}\right)=\frac{1}{3}\left(2 \mathrm{C}_{i j}^{k l}+\mathrm{C}_{i j}^{k l}\right)=\mathrm{C}_{i j}^{k l},
$$

where equation (42) has been used once again. Therefore $\phi \circ \varphi=\operatorname{Id}_{\Sigma}$ and, being both vector spaces of the same dimension, it turns out that $\phi$ and $\varphi$ are isomorphisms and $\phi=\varphi^{-1}$, and so equation (35) has been proved.

\section{Linear computation of the QoS}

Each segment $\{\mathbf{x}, \mathbf{y}\}$ of length $d$ provides a linear equation on $\mathbf{C}$

$$
\sigma(\mathbf{x}, \mathbf{y})^{\top} \mathrm{C} \sigma(\mathbf{x}, \mathbf{y})=0 .
$$

By result (26), we know that

$$
L \stackrel{\text { def }}{=} \operatorname{dim} \operatorname{Sym}(N+1)-1=(N+1)(N+2) / 2-1
$$

equations are required to determine $\mathrm{C}$, since there is no linear subspace of lower dimension where the quadrics $\mathrm{C}$ lie. Moreover, result 5.4 assures that the unique solution $\mathrm{C}$ can be obtained using $L$ segments of length $d$ in general position.

Let us now address the problem of recovering the Euclidean geometry from the knowledge of the arbitrary lengths $d_{i}$ of a set of segments $\left\{\mathbf{x}_{i}, \mathbf{y}_{i}\right\}$. Since the corresponding quadrics $\mathrm{C}_{d_{i}}$ are written as

$$
\mathrm{C}_{d_{i}}=\mathrm{C}_{1}+\frac{d_{i}^{2}}{2} \mathrm{C}_{2}
$$

it is enough to obtain $\mathrm{C}_{1}$ and $\mathrm{C}_{2}$ from the linear equations

$$
\sigma\left(\mathbf{x}_{i}, \mathbf{y}_{i}\right)^{\top}\left(\mathbf{C}_{1}+\frac{d_{i}^{2}}{2} \mathrm{C}_{2}\right) \sigma\left(\mathbf{x}_{i}, \mathbf{y}_{i}\right)=0
$$

which, in principle, amounts to computing $2 L+1$ parameters up to a common scale factor. However we next see how, due to result $6.1, L$ linear equations suffice.

From results 5.3 and 6.1 we already know that $S_{2}$ is the linear space generated by matrices of the form $\sigma(\mathbf{x}, \mathbf{x}) \sigma(\mathbf{x}, \mathbf{x})^{\top}$. Therefore a basis $\left\{\mathrm{M}_{\beta}^{(2)}\right\}_{\beta=1}^{\operatorname{dim} S_{2}}$ of $S_{2}$ can be readily obtained from this parametrization. Again from result 6.1, $S_{1}=S_{2}^{\perp}$, therefore a basis $\left\{\mathrm{M}_{\alpha}^{(1)}\right\}_{\alpha=1}^{\operatorname{dim} S_{1}}$ of $S_{1}$ can be obtained solving for $\mathrm{C}_{1}$ the system of $\operatorname{dim} S_{2}$ linear equations

$$
\left\langle\mathrm{M}_{\beta}^{(2)}, \mathrm{C}_{1}\right\rangle=0 \text {. }
$$

Once obtained these bases, we can parametrize linearly $\mathrm{C}_{1}$ and $\mathrm{C}_{2}$ as follows:

$$
\begin{aligned}
& \mathrm{C}_{1}=\sum_{\alpha=1}^{\operatorname{dim} S_{1}} a_{\alpha}^{(1)} \mathrm{M}_{\alpha}^{(1)} \\
& \mathrm{C}_{2}=\sum_{\beta=1}^{\operatorname{dim} S_{2}} a_{\beta}^{(2)} \mathrm{M}_{\beta}^{(2)}
\end{aligned}
$$

for some unknown coefficients $a_{\alpha}^{(1)}, a_{\beta}^{(2)}$. Now equations (45) can be written as

$$
\sum_{\alpha=1}^{\operatorname{dim} S_{1}} \mu_{\alpha}^{i} a_{\alpha}^{(1)}+\frac{d_{i}^{2}}{2} \sum_{k=1}^{\operatorname{dim} S_{2}} \nu_{\beta}^{i} a_{\beta}^{(2)}=0
$$


where

$$
\begin{aligned}
\mu_{\alpha}^{i} & =\sigma\left(\mathbf{x}_{i}, \mathbf{y}_{i}\right)^{\top} \mathbf{M}_{\alpha}^{(1)} \sigma\left(\mathbf{x}_{i}, \mathbf{y}_{i}\right), \\
\nu_{\beta}^{i} & =\sigma\left(\mathbf{x}_{i}, \mathbf{y}_{i}\right)^{\top} \mathbf{M}_{\beta}^{(2)} \sigma\left(\mathbf{x}_{i}, \mathbf{y}_{i}\right) .
\end{aligned}
$$

Therefore from these $\operatorname{dim} S_{1}+\operatorname{dim} S_{2}-1=L$ equations the $L+1$ unknowns $a_{j}^{(1)}, a_{k}^{(2)}$ can be recovered up to a common scale factor and thus the QoS is recovered.

\section{EXPERIMENTAL RESULTS}

10.1. General setting. The performances of the algorithms suggested by the theory presented in this paper have been evaluated in different experimental settings. Some of the implementations make use of affine adjustment. Four algorithms have been considered:

- Extraction of the rectifying homography from $\mathrm{C}_{1}(\mathrm{C} 1)$.

- Extraction of the rectifying homography from $\mathrm{C}_{1}$ followed by affine adjustment $(\mathrm{C} 1 \mathrm{~A})$.

- Affine calibration using the plane at infinity extracted from $\mathrm{C}_{2}$ followed by affine adjustment (C2A).

- Pure affine adjustment (A) (included just for comparison).

The experiments are based on sets of equal-length segments, so the main quality parameter computed for each reconstruction is the typical deviation of the corrected segment lengths, divided by its average $(\sigma / \mu$ parameter $)$. The failure probabilities of the algorithms have also been studied: besides the possible failure of affine adjustment (if the computed approximation of $U^{\top} U$ is not definite (2)) the extraction of the rectifying homography from $\mathrm{C}_{1}$ fails if the obtained rank-three $\mathrm{AQC}$ matrix is not semidefinite.

10.2. Experiments with synthetic data. The first set of experiments deal with $2 \mathrm{D}$ data. Random segments of unit length are obtained within a square with corners $\mathbf{v}_{i}, i=$ $1,2,3,4$, and a random homography is applied to them. The homography is defined as the one that maps each point $\mathbf{v}_{i}$ to $\mathbf{v}_{i}+\mathbf{n}_{i}$, where $\mathbf{n}_{i}$ is a random vector of independent zero-mean gaussian components of typical deviation $\sigma 1 / 8$ of the square side. Then zeromean gaussian noise of independent components is added to the distorted points and the algorithms are applied to obtain the rectifying homography using the original segment lengths as input. Figure 1 shows a set of sample data.

The algorithms have been tested in a similar way in the 3D case, employing random segments of unit length with Euclidean coordinates in a cube of vertices $\mathbf{v}_{i}, i=1, \ldots, 8$. The random homography is defined as the one that maps five selected vertices of the cube to the corresponging points $\mathbf{v}_{i}+\mathbf{n}_{i}$ with $\mathbf{n}_{i}$ defined as in the $2 \mathrm{D}$ case. Figure 2 shows a set of sample data.

Figure 3 shows for 2D and 3D, respectively, the performances of the different algorithms as a function of the noise variance. The dependence of the algorithms performance with the number of segments is illustrated in figure 4. Algorithm C1A can be seen to be the best or to perform very close to the best in terms of $\sigma / \mu$ quality parameter, while the advantage of $\mathrm{C} 2 \mathrm{~A}$ over $\mathrm{C} 1 \mathrm{~S}$ obsrved in $2 \mathrm{D}$ reverts in the $3 \mathrm{D}$ case. Algorithm $\mathrm{C} 1 \mathrm{~A}$ also performs well in terms of failure rate, while $\mathrm{C} 2 \mathrm{~A}$ is uniformly the worst in terms of this parameter.

In figure 4 the comparison of the three first algorithms with the reference algorithm $\mathrm{C} 2 \mathrm{~A}$ reveals that their performance is very poor when the number of segments is the 

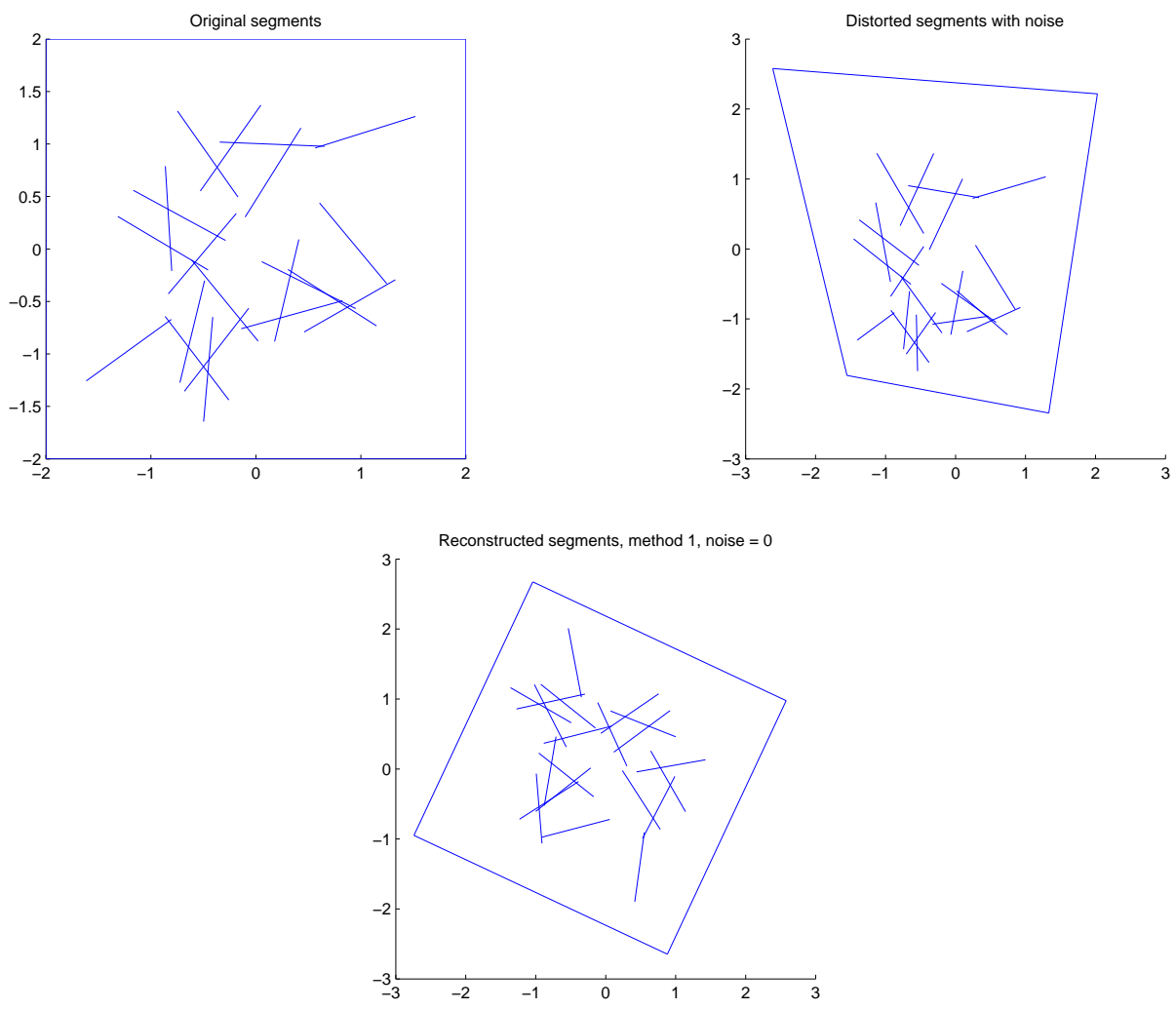

Figure 1. 2D synthetic data: sample data

minimum necessary. However, the performances improve considerably when the number of segments is doubled and from then on the performances improve slowly.

The second experiment with synthetic data addresses the Euclidean upgrading of projective reconstructions obtained from images. For each instance of the experiment a set of random 3D segments of equal length inside a cube are generated, together with random intrinsic and extrinsic parameters of two cameras, oriented so that the cube appears approximately in the center of the image, with points projecting up to 1000 pixels far from the center of the image. Zero-mean gaussian noise of independent components is added to these projections. One of these random configurations is shown in figure 5. A projective reconstruction of the scene is obtained from the two images using algorithms 10.1 (eight-point algorithm to compute the fundamental matrix) and 11.1 (optimal triangulation) of [5], which is then made quasi-affine using algorithm 20.1 of [5]. The four tested techniques are then applied. The results, in figure 6, are similar to those in the previous set of experiments.

10.3. Experiments with real images. In order to test the performance of the algorithms for the $2 \mathrm{D}$ case with real data, a picture of a sheet of paper showing random segments of the same length was taken. After detecting the segment ends, rectifying homographies have been obtained using the four algorithms tested. The results are shown in figure 7. Particularly satisfactory is the $\sigma / \mu$ value, equal to 0.014 , obtained using algorithm $\mathrm{C} 2 \mathrm{~A}$.

The experiments of 3D reconstruction based on real images have been performed using a bar with three equidistant LEDs, whose motion is recorded with two synchronized video 

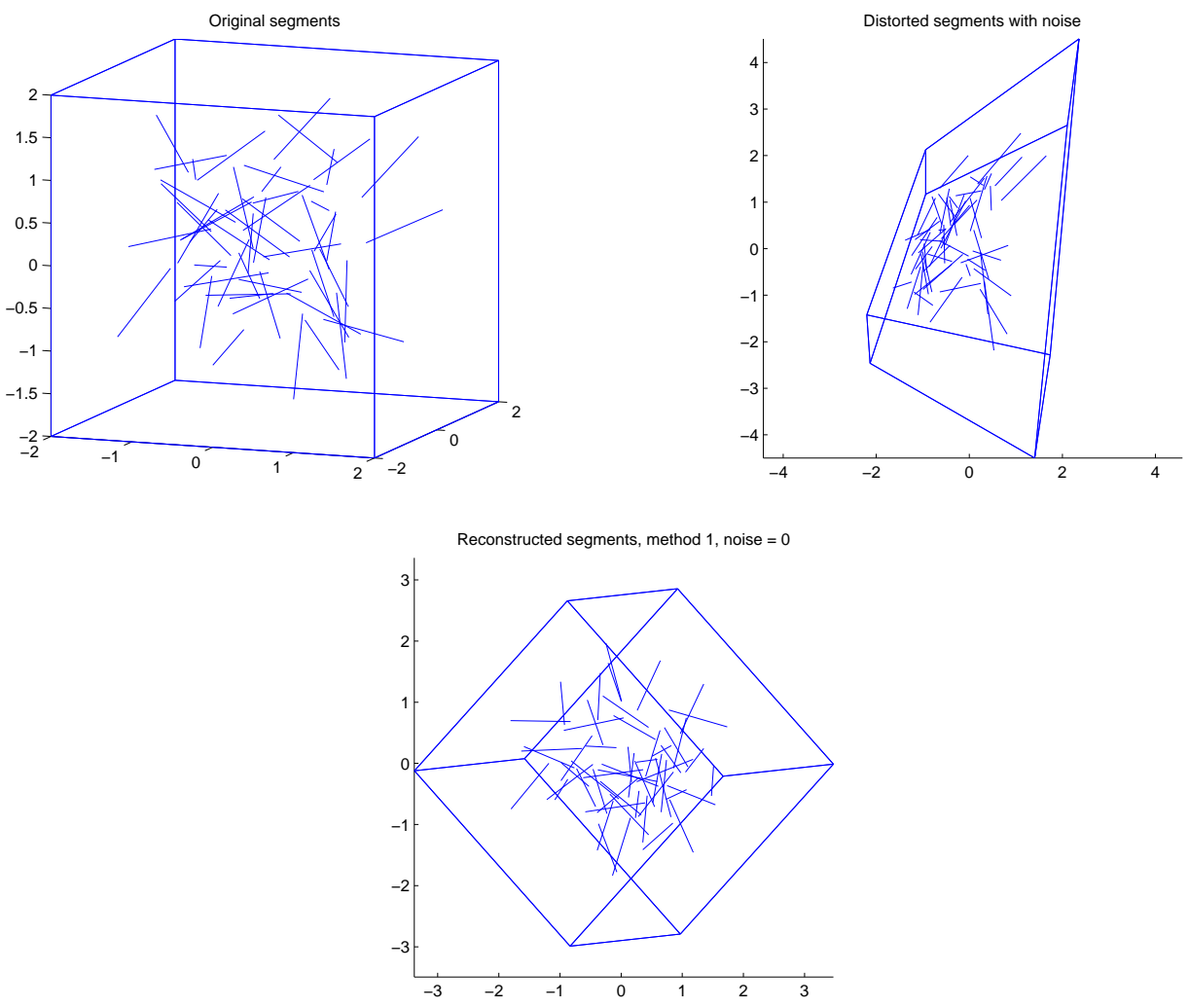

Figure 2. 3D synthetic data: sample data

cameras with a resolution of $1280 \times 960$ pixels. The detection of the LEDs is performed automatically, and a projective reconstruction is obtained which is then upgraded to a quasi-affine reconstruction. The Euclidean upgrading is performed with the proposed algorithms, employing exclusively the knowledge of the distance between the two LEDs at the extremes of the bar, the LED in the middle only being used only to provide an additional measure of the accuracy of the reconstruction.

Figure 8 shows the detected points for 131 valid frames and the resulting 3D reconstruction with method $\mathrm{C} 2 \mathrm{~A}$. In this reconstruction the maximum length is a $4 \%$ larger than the minimum length. The ratios of the distances between the LED in the middle and the LEDs at each extreme of the bar show and average value of 0.992 and a maximum value an $8 \%$ larger than the minimum one.

\section{Appendix A. TeChnical Details}

A.1. Proof of result 5.4. In order to prove result 5.4 we need the following two lemmas:

Lemma A.1. Let $\mathrm{C}$ and $\mathrm{C}^{\prime}$ be two quadrics of $\mathbf{P}^{N}$ taking the same values along $\operatorname{im} \sigma$, i.e.,

$$
\sigma(\mathbf{x}, \mathbf{y})^{\top} \mathbf{C} \sigma(\mathbf{x}, \mathbf{y})=\sigma(\mathbf{x}, \mathbf{y})^{\top} \mathbf{C}^{\prime} \sigma(\mathbf{x}, \mathbf{y})
$$

for any $\mathbf{x}, \mathbf{y} \in \mathbf{P}^{N}$. Then $\mathbf{C}=\mathbf{C}^{\prime}$.

Proof. Writing our hypothesis as

$$
\sigma(\mathbf{x}, \mathbf{y})^{\top}\left(\mathbf{C}-\mathbf{C}^{\prime}\right) \sigma(\mathbf{x}, \mathbf{y})=0
$$



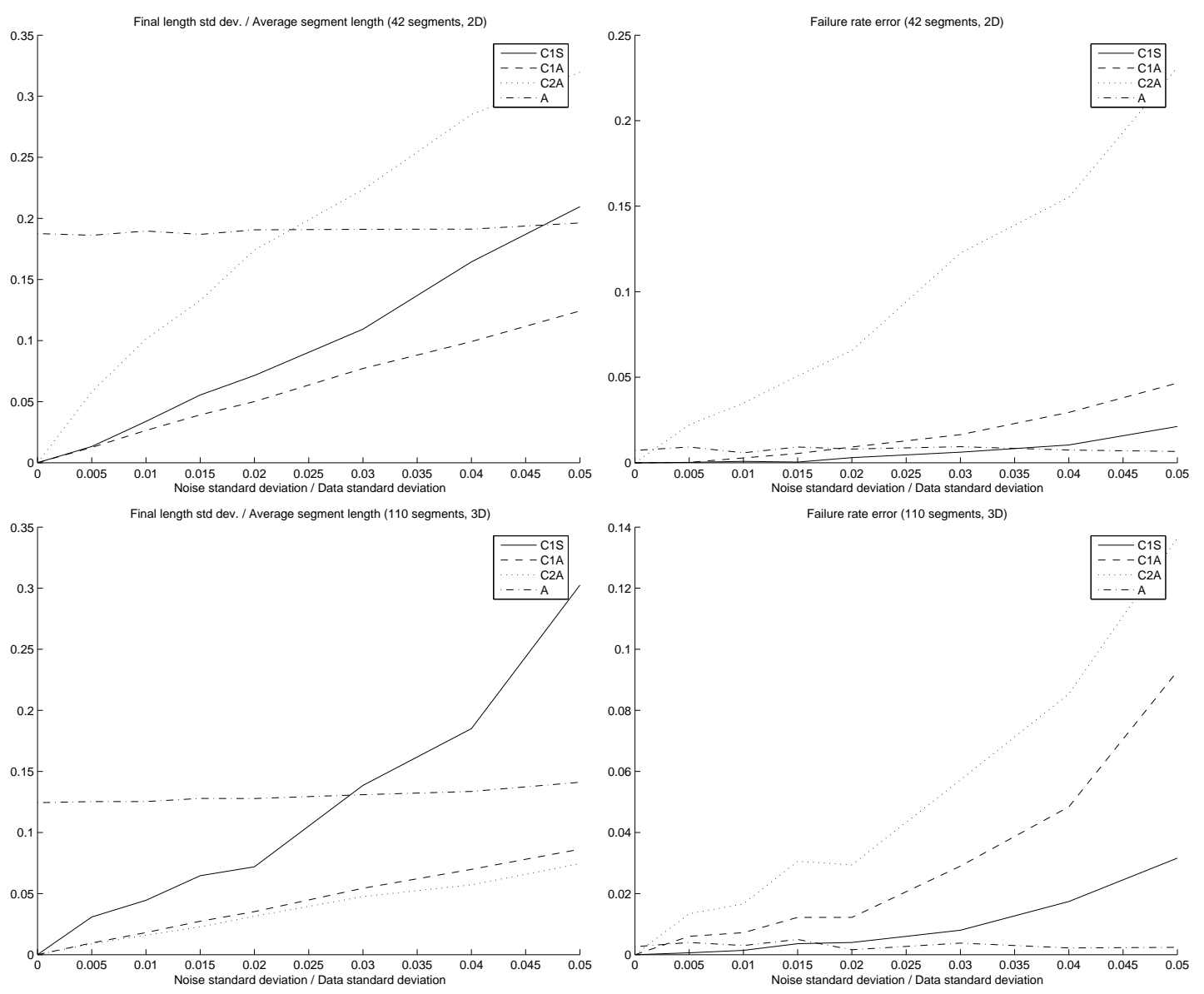

Figure 3. Synthetic data: Dependence of the performance with noise variance for 2D (top) and 3D (bottom) for 42 and 110 segments respectively.

we see that it is enough to prove that $\sigma(\mathbf{x}, \mathbf{y})^{\top} \mathbf{C} \sigma(\mathbf{x}, \mathbf{y})=0$ for any $\mathbf{x}, \mathbf{y}$ implies $\mathbf{C}=0$. Let us denote

$$
E=\sigma(\mathbf{x}, \mathbf{y})^{\top} \mathrm{C} \sigma(\mathbf{x}, \mathbf{y}) .
$$

Let us denote by $\left\{\mathbf{e}_{i}\right\}$ the canonical basis and let us define

$$
\mathrm{C}_{i j}^{k l}=\sigma\left(\mathbf{e}_{i}, \mathbf{e}_{j}\right)^{\top} \mathrm{C} \sigma\left(\mathbf{e}_{k}, \mathbf{e}_{l}\right),
$$

we see that the coefficients $\mathrm{C}_{i j}^{k l}$ satisfy the symmetries

$$
\mathrm{C}_{i j}^{k l}=\mathrm{C}_{k l}^{i j}=\mathrm{C}_{i j}^{l k}=\mathrm{C}_{j i}^{k l},
$$

It is easy to compute the 4 th derivative

$$
\frac{\partial^{4} E}{\partial x_{i} \partial y_{j} \partial x_{k} \partial y_{l}}=2\left(\sigma\left(\mathbf{e}_{i}, \mathbf{e}_{j}\right)^{\top} \mathrm{C} \sigma\left(\mathbf{e}_{k}, \mathbf{e}_{l}\right)+\sigma\left(\mathbf{e}_{i}, \mathbf{e}_{l}\right)^{\top} \mathrm{C} \sigma\left(\mathbf{e}_{k}, \mathbf{e}_{j}\right)\right)=0,
$$

since $E=0$, and therefore we have the additional symmetry

$$
\mathrm{C}_{i j}^{k l}=-\mathrm{C}_{i l}^{k j}
$$

so that

$$
\mathrm{C}_{i j}^{k l}=-\mathrm{C}_{i l}^{j k}=\mathrm{C}_{i k}^{j l}=-\mathrm{C}_{i j}^{l k}=-\mathrm{C}_{i j}^{k l},
$$

and so $\sigma\left(\mathbf{e}_{i}, \mathbf{e}_{j}\right)^{\top} \mathrm{C} \sigma\left(\mathbf{e}_{k}, \mathbf{e}_{l}\right)=\mathrm{C}_{i j}^{k l}=0$ for any $i, j, k$ and $l$. Since $\left\{\sigma\left(\mathbf{e}_{i}, \mathbf{e}_{j}\right): i \leq j\right\}$ is a basis of $\mathbf{C}^{N+1}$, we conclude that $\mathrm{C}=0$. 

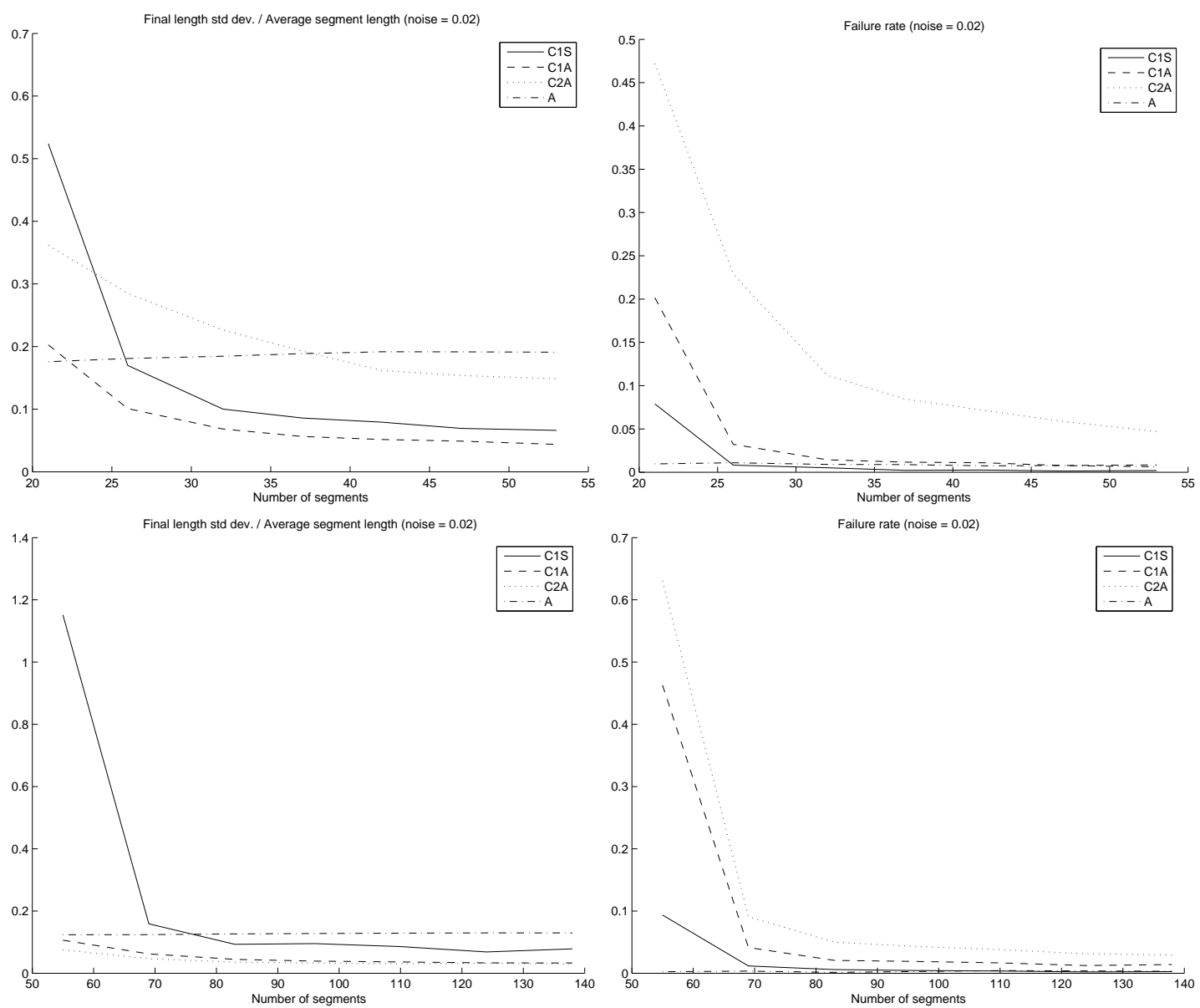

Figure 4. Synthetic data: Dependence of the performance with the number of segments for 2D (top) and 3D (bottom) for noise typical deviation $2 \%$ of data typical deviation.

Lemma A.2. If all the irreducible factors of

$$
E=\sigma(\mathbf{x}, \mathbf{y})^{\top} \mathrm{C} \sigma(\mathbf{x}, \mathbf{y}) .
$$

are of degree one then $E$ is of the form

$$
E(\mathbf{x}, \mathbf{y})=\left(\mathbf{a}^{\top} \mathbf{x}\right)\left(\mathbf{a}^{\top} \mathbf{y}\right)\left(\mathbf{b}^{\top} \mathbf{x}\right)\left(\mathbf{b}^{\top} \mathbf{y}\right)
$$

for some $\mathbf{a}, \mathbf{b} \in \mathbf{C}^{n+1}$.

Proof. Let $E(\mathbf{x}, \mathbf{y})=\left(\mathbf{a}^{\top} \mathbf{x}+\mathbf{a}^{\prime \top} \mathbf{y}\right)\left(\mathbf{b}^{\top} \mathbf{x}+\mathbf{b}^{\prime \top} \mathbf{y}\right)\left(\mathbf{c}^{\top} \mathbf{x}+\mathbf{c}^{\prime \top} \mathbf{y}\right)\left(\mathbf{d}^{\top} \mathbf{x}+\mathbf{d}^{\prime \top} \mathbf{y}\right)$. From the fact that all the monomials of $E$ are of degree two both in the variables $x_{i}$ and $y_{i}$ we deduce that $E$ must be of the form $E(\mathbf{x}, \mathbf{y})=\left(\mathbf{a}^{\top} \mathbf{x}\right)\left(\mathbf{b}^{\top} \mathbf{x}\right)\left(\mathbf{c}^{\top} \mathbf{y}\right)\left(\mathbf{d}^{\top} \mathbf{y}\right)$ and taking into account the symmetry $E(\mathbf{x}, \mathbf{y})=E(\mathbf{y}, \mathbf{x})$ it follows that $E$ must have the required form.

Now we are in conditions to give a proof of result 5.4.

Proof. Let us denote

$$
E(\mathbf{x}, \mathbf{y})=\sigma(\mathbf{x}, \mathbf{y})^{\top} \mathbf{C} \sigma(\mathbf{x}, \mathbf{y})
$$

and

$$
E^{\prime}(\mathbf{x}, \mathbf{y})=\sigma(\mathbf{x}, \mathbf{y})^{\top} \mathbf{C}^{\prime} \sigma(\mathbf{x}, \mathbf{y})
$$



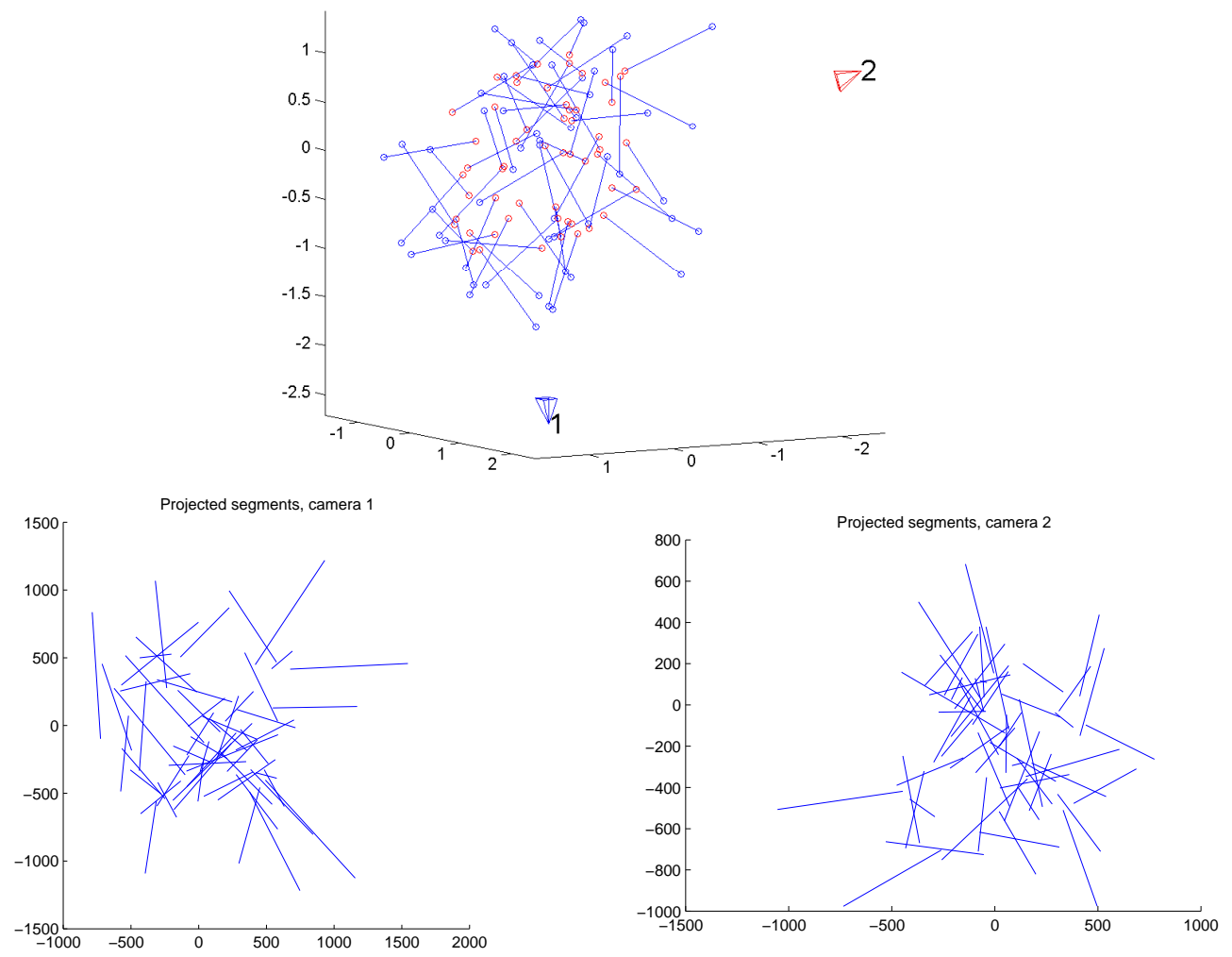

Figure 5. Synthetic data: Reconstruction from images. Top: An instance of original synthetic scene. Bottom: Corresponding views of the segments.
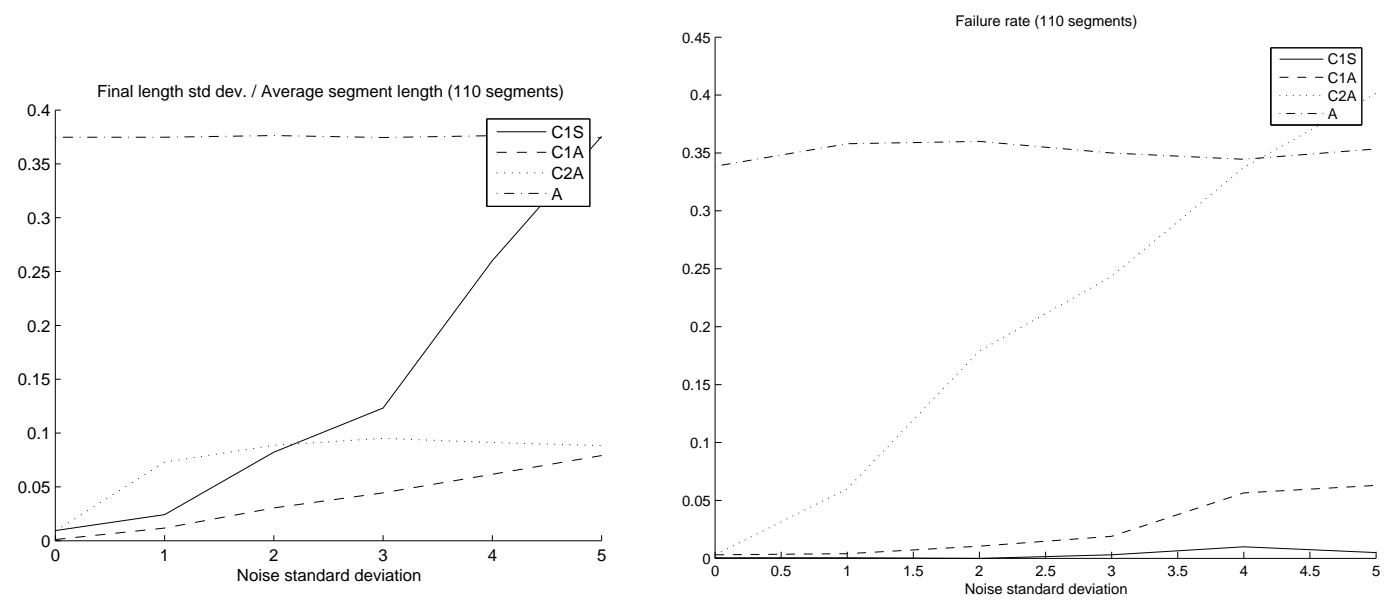

Figure 6. Synthetic data: Reconstruction from images. Algorithms performance as a function of the image noise.

and let

$$
\begin{aligned}
E & =\left(E_{1}\right)^{a_{1}} \cdots\left(E_{s}\right)^{a_{s},}, \\
E^{\prime} & =\left(E_{1}^{\prime}\right)^{a_{1}^{\prime}} \cdots\left(E_{s^{\prime}}^{\prime}\right)^{a_{s^{\prime}}^{\prime},}
\end{aligned}
$$



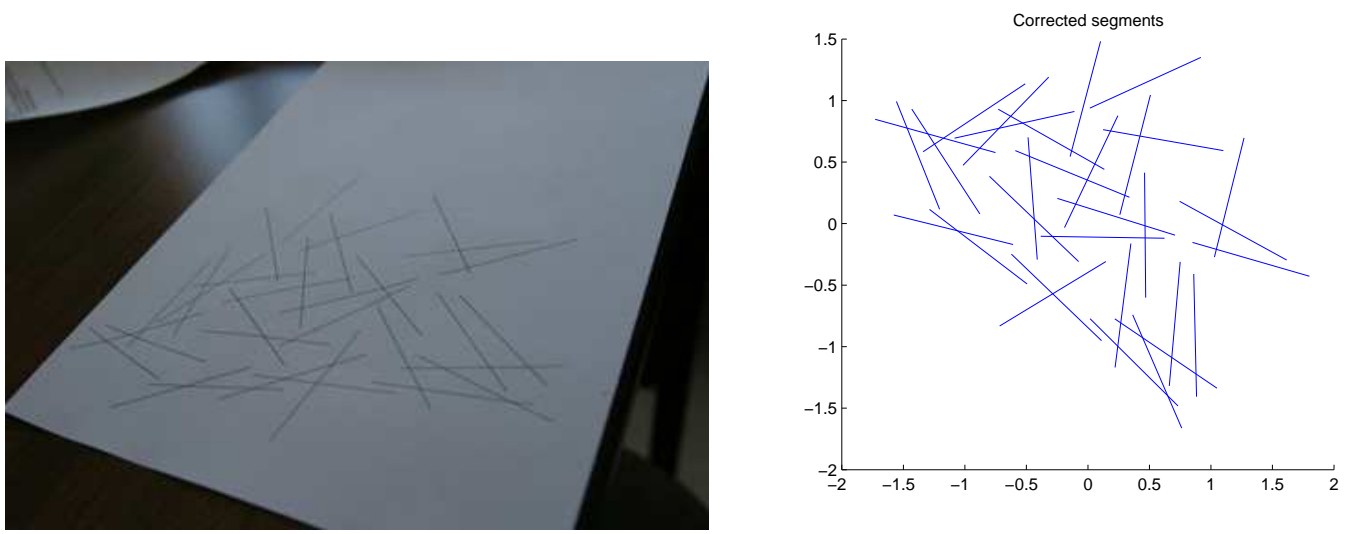

Figure 7. Experiments with 2D real data. Original image (left) and its corrected version (right).
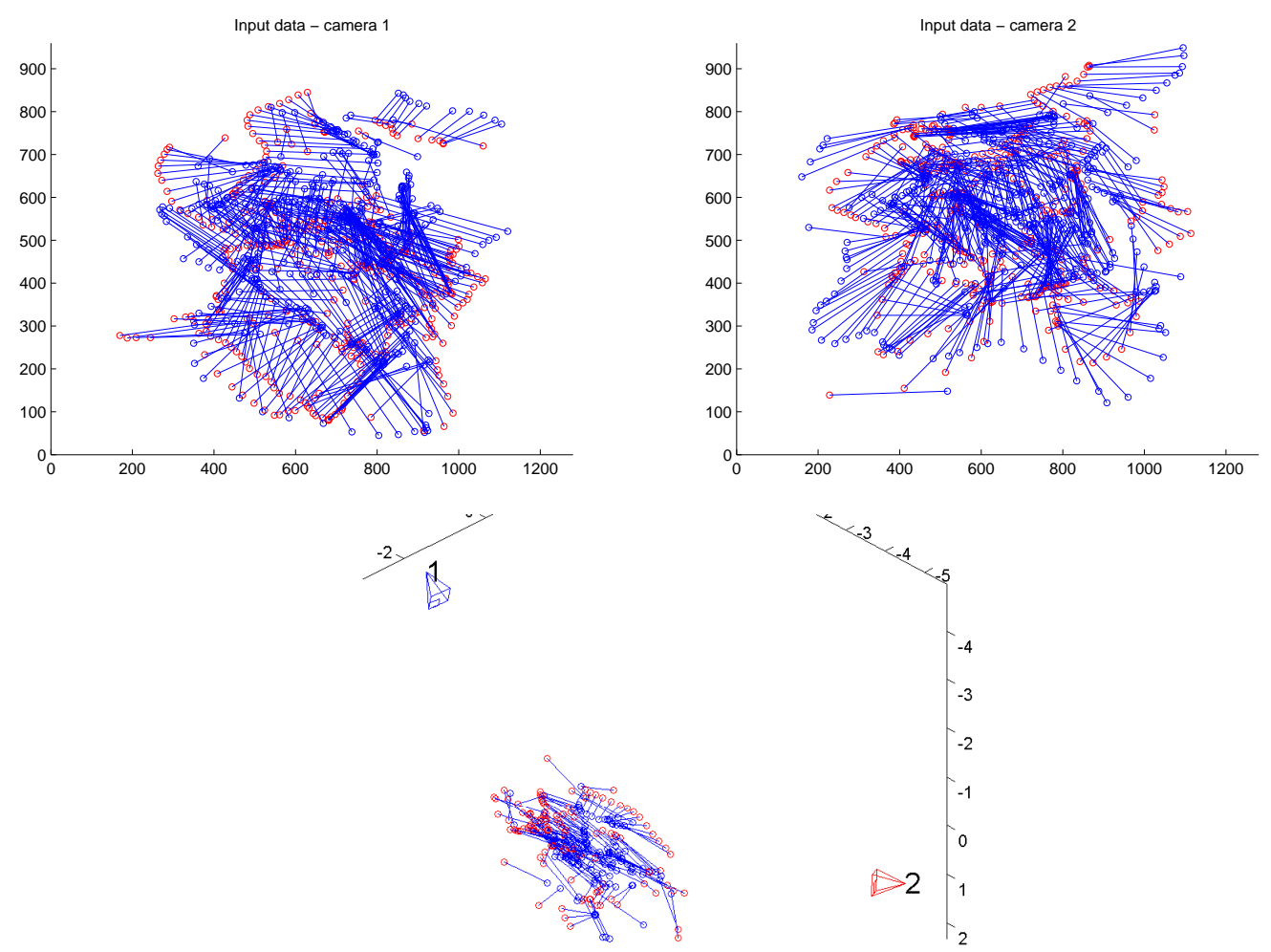

Figure 8. Experiments of 3D reconstruction with real data. Views of the moving segment with both cameras (top) and reconstructed 3D scene (bottom).

be their decomposition in irreducible factors. Since $E(\mathbf{x}, \mathbf{y})$ and $E^{\prime}(\mathbf{x}, \mathbf{y})$ have the same zeros, they have the same reduced polynomial $R$ as a consequence of Hilbert's Nullstellensatz [3, p.49], i.e.,

$$
R=E_{1} \cdots E_{s}=E_{1}^{\prime} \cdots E_{s^{\prime}}^{\prime}
$$


up to a constant scale factor $\lambda$ which we assume without loss of generality to be $\lambda=1$. Sorting factors if necessary, we obtain $s=s^{\prime}$ and $E_{i}=E_{i}^{\prime}$ for any $i=1, \ldots, s$. We have several possibilities according to whether $E$ is reduced or not:

- First, if $E$ is reduced, i.e., $E=R$ then it follows that $E^{\prime}=R$ too, for if any $a_{i}^{\prime}>1$ then $\operatorname{deg} E^{\prime}>\operatorname{deg} E$, which is impossible. Now, being $E=E^{\prime}$ the result follows from lemma A.1.

- If $E$ is not reduced, it is easy to check that the following are the only possibilities (taking into account that $\operatorname{deg} E=\operatorname{deg} E^{\prime}=4$ ). Let us denote $E_{i}=E_{i}^{(d)}$ whenever $\operatorname{deg} E_{i}=d$ :

(1) If $E=\left(E_{1}^{(2)}\right)^{2}$ comparison of irreducible factors readily leads us to $E^{\prime}=$ $\left(E_{1}^{(2)}\right)^{2}$ and so $E=E^{\prime}$ and the result follows.

(2) Similarly, if $E=E_{1}^{(2)}\left(E_{2}^{(1)}\right)^{2}$ then necessarily also $E=E^{\prime}$.

(3) If all the irreducible components are of degree one then, taking into account lemma A.2, since $E$ is not reduced the only possibility is that $\mathbf{a}=\mathbf{b}$ and so

$$
E(\mathbf{x}, \mathbf{y})=\left(\mathbf{a}^{\top} \mathbf{x}\right)^{2}\left(\mathbf{a}^{\top} \mathbf{y}\right)^{2} .
$$

But in this case, matching of irreducible components also forces $E=E^{\prime}$ and so the result is proved.

\section{A.2. Lemmas needed for the proof of result 6.1 .}

\section{Lemma A.3.}

$$
\operatorname{dim} \operatorname{Span}\left\{\mathrm{E}^{1}(\mathrm{H}): \mathrm{H} \in \mathbf{M}_{n+1}\right\} \geq \frac{1}{12} n(n+1)^{2}(n+2) .
$$

Proof. To do so, let us define

$$
\mathrm{E}_{i j k l}^{1}=\frac{\partial^{4} \mathrm{E}^{1}}{\partial h_{i 0} \partial h_{j 0} \partial h_{k 1} \partial h_{l 1}} .
$$

Since all the derivatives $\mathrm{E}_{i j k l}^{1} \in \operatorname{Span}\left\{\mathrm{E}^{1}(\mathrm{H}): \mathrm{H} \in \mathbf{M}_{n+1}\right\}$, we have that

$$
\operatorname{dim} \operatorname{Span}\left\{\mathrm{E}^{1}(\mathrm{H}): \mathrm{H} \in \mathbf{M}_{n+1}\right\} \geq \operatorname{dim} \operatorname{Span}\left\{\mathrm{E}_{i j k l}^{1}(\mathrm{H}): \mathrm{H} \in \mathbf{M}_{n+1}, 0 \leq i, j, k, l \leq n+1\right\} .
$$

It is not hard to check that

$$
\mathrm{E}_{i j k l}^{1}=-2 \mathrm{~S}\left(\sigma\left(\mathbf{e}_{i}, \mathbf{e}_{j}\right), \sigma\left(\mathbf{e}_{k}, \mathbf{e}_{l}\right)\right)+\mathrm{S}\left(\sigma\left(\mathbf{e}_{i}, \mathbf{e}_{k}\right), \sigma\left(\mathbf{e}_{j}, \mathbf{e}_{l}\right)\right)+\mathrm{S}\left(\sigma\left(\mathbf{e}_{i}, \mathbf{e}_{l}\right), \sigma\left(\mathbf{e}_{j}, \mathbf{e}_{k}\right)\right),
$$

where $\mathbf{S}$ is defined as in (1). The following lemma assures that the matrices $\mathrm{E}_{i j k l}^{1}$ are linearly independent:

Lemma A.4. Two matrices $\mathrm{E}_{i j k l}^{1}$ and $\mathrm{E}_{i^{\prime} j^{\prime} k^{\prime} l^{\prime}}^{1}$ are orthogonal whenever $\{i, j, k, l\} \neq\left\{i^{\prime}, j^{\prime}, k^{\prime}, l^{\prime}\right\}$.

Proof. This is straightforward consequence of the fact that the products

$$
\left\langle\mathbf { S } \left(\sigma\left(\mathbf{e}_{i}, \mathbf{e}_{j}\right), \sigma\left(\mathbf{e}_{k}, \mathbf{e}_{l}\right), \mathbf{S}\left(\sigma\left(\mathbf{e}_{i^{\prime}}, \mathbf{e}_{j^{\prime}}\right), \sigma\left(\mathbf{e}_{k^{\prime}}, \mathbf{e}_{l^{\prime}}\right)\right\rangle\right.\right.
$$

vanish whenever $\{i, j, k, l\} \neq\left\{i^{\prime}, j^{\prime}, k^{\prime}, l^{\prime}\right\}$.

Let us denote by $\mathcal{P}_{r}(n+1)$ the subset of the power set of $\{0, \ldots, n\}$ given by the subsets of cardinal $r$. For each element $\alpha \in \mathcal{P}_{r}(n+1)$, which is an unordered set, let us choose an ordering given by a concrete $r$-sequence. We denote such sequence by $s^{\alpha}=\left(s_{1}^{\alpha}, \ldots, s_{r}^{\alpha}\right)$, and we have $\left\{s_{1}^{\alpha}, \ldots, s_{r}^{\alpha}\right\}=\alpha$. Let $\mathcal{S}_{r}$ be the set of all such sequences, one for each $\alpha$. 
For each $s=(i, j, k, l) \in \mathcal{S}_{4}$ we define the vector subspace $V_{s}^{(2)}$ spanned by $\mathrm{E}_{i j k l}^{1}$ and $\mathrm{E}_{i k j l}^{1}$. In addition, we define for each $s=(i, j, k) \in \mathcal{S}_{3}$ the vector subspace $V_{s}^{(3)}$ spanned by $\mathrm{E}_{i i j k}^{1}, \mathrm{E}_{i j j k}^{1}$ and $\mathrm{E}_{i j k k}^{1}$. Finally, if $s=(i, j) \in \mathcal{S}_{2}$ we define $V_{s}^{(1)}$ as the vector subspace spanned by $\mathrm{E}_{i i j j}^{1}$.

Lemma A.5. The dimension of each $V_{s}^{(h)}$ is $h$.

Proof. Let us check that $\operatorname{dim} V_{s}^{(2)}=2$, the other cases being treated similarly. Denoting $s=(i, j, k, l)$,

$$
\begin{aligned}
& \mathrm{E}_{i j k l}^{1}=-2 \mathrm{~S}\left(\sigma\left(\mathbf{e}_{i}, \mathbf{e}_{j}\right), \sigma\left(\mathbf{e}_{k}, \mathbf{e}_{l}\right)\right)+\mathrm{S}\left(\sigma\left(\mathbf{e}_{i}, \mathbf{e}_{k}\right), \sigma\left(\mathbf{e}_{j}, \mathbf{e}_{l}\right)\right)+\mathrm{S}\left(\sigma\left(\mathbf{e}_{i}, \mathbf{e}_{l}\right), \sigma\left(\mathbf{e}_{j}, \mathbf{e}_{k}\right)\right), \\
& \mathrm{E}_{i k j l}^{1}=-2 \mathrm{~S}\left(\sigma\left(\mathbf{e}_{i}, \mathbf{e}_{k}\right), \sigma\left(\mathbf{e}_{j}, \mathbf{e}_{l}\right)\right)+\mathrm{S}\left(\sigma\left(\mathbf{e}_{i}, \mathbf{e}_{j}\right), \sigma\left(\mathbf{e}_{k}, \mathbf{e}_{l}\right)\right)+\mathrm{S}\left(\sigma\left(\mathbf{e}_{i}, \mathbf{e}_{l}\right), \sigma\left(\mathbf{e}_{k}, \mathbf{e}_{j}\right)\right),
\end{aligned}
$$

Being $\sigma\left(\mathbf{e}_{i}, \mathbf{e}_{j}\right), \sigma\left(\mathbf{e}_{k}, \mathbf{e}_{l}\right), \sigma\left(\mathbf{e}_{i}, \mathbf{e}_{k}\right), \sigma\left(\mathbf{e}_{j}, \mathbf{e}_{l}\right), \sigma\left(\mathbf{e}_{i}, \mathbf{e}_{l}\right), \sigma\left(\mathbf{e}_{j}, \mathbf{e}_{k}\right)$ six independent vectors, since they are proportional to different vectors of the canonical basis of $\mathbf{C}^{N+1}$, the symmetrized matrices $\mathbf{S}\left(\sigma\left(\mathbf{e}_{i}, \mathbf{e}_{j}\right), \sigma\left(\mathbf{e}_{k}, \mathbf{e}_{l}\right)\right), \mathbf{S}\left(\sigma\left(\mathbf{e}_{i}, \mathbf{e}_{k}\right), \sigma\left(\mathbf{e}_{j}, \mathbf{e}_{l}\right)\right), \mathbf{S}\left(\sigma\left(\mathbf{e}_{i}, \mathbf{e}_{l}\right), \sigma\left(\mathbf{e}_{j}, \mathbf{e}_{k}\right)\right)$ are also independent. Being the coordinates of $\mathrm{E}_{i j k l}^{1}$ and $\mathrm{E}_{i k j l}^{1}$ in terms of the basis formed by these three matrices $(-2,1,1)$ and $(1,-2,1)$, they are linearly independent, i.e., $\operatorname{dim} V_{s}^{(2)}=2$.

Now let us consider the sum of vector subspaces

$$
\bigoplus_{s \in \mathcal{S}_{4}} V_{s}^{(2)} \oplus \bigoplus_{s \in \mathcal{S}_{3}} V_{s}^{(3)} \oplus \bigoplus_{s \in \mathcal{S}_{2}} V_{s}^{(1)}
$$

Due to lemma A.4 this is an orthogonal sum and its dimension is, due to lemma A.5

$$
2\left(\begin{array}{c}
n+1 \\
4
\end{array}\right)+3\left(\begin{array}{c}
n+1 \\
3
\end{array}\right)+\left(\begin{array}{c}
n+1 \\
2
\end{array}\right)=\frac{1}{12} n(n+1)^{2}(n+2) .
$$

This proves that

$$
\operatorname{dim} S_{1} \geq \frac{1}{12} n(n+1)^{2}(n+2) .
$$

which together with $(29)$ proves that $\operatorname{dim} S_{1}=\frac{1}{12} n(n+1)^{2}(n+2)$.

\section{REFERENCES}

[1] M. Agrawal and L. S. Davis. Camera calibration using spheres: A semi-definite programming approach. In ICCV '03: Proceedings of the Ninth IEEE International Conference on Computer Vision, page 782, Washington, DC, USA, 2003. IEEE Computer Society.

[2] E. Bayro-Corrochano and B. Rosenhahn. A geometric approach for the analysis and computation of the intrinsic camera parameters. Pattern Recognition, 35(1):169 - 186, 2002.

[3] J. Harris. Algebraic Geometry, A First Course. Springer, 1995.

[4] R. Hartley and A. Zisserman. Multiple View Geometry in Computer Vision. Cambridge University Press, second edition, 2003.

[5] R. I. Hartley. Estimation of relative camera positions for uncalibrated cameras. In Proc. European Conference on Computer Vision, pages 579-587, London, UK, 1992. Springer-Verlag.

[6] R. I. Hartley, E. Hayman, L. de Agapito, and I. Reid. Camera calibration and the search for infinity. Computer Vision, IEEE International Conference on, 1:510, 1999.

[7] A. Heyden and K. Åström. Euclidean reconstruction from image sequences with varying and unknown focal length and principal point. In Proc. IEEE Conference on Computer Vision and Pattern Recognition, New York, USA, 1997.

[8] F. Kahl, B. Triggs, and K. Åström. Critical motions for auto-calibration when some intrinsic parameters can vary. Journal of Mathematical Imaging and Vision, 13(2):131-146, 2000. 
[9] D. Liebowitz and S. Carlsson. Uncalibrated motion capture exploiting articulated structure constraints. Int. J. Comput. Vision, 51(3):171-187, 2003.

[10] S. J. Maybank and O. D. Faugeras. A theory of self-calibration of a moving camera. Int. J. Comput. Vision, 8(2):123-151, 1992.

[11] M. Pollefeys and L. V. Gool. A stratified approach to metric self-calibration. In Proc. of the IEEE Conference on Computer Vision and Pattern Recognition, pages 407-412, June 1997.

[12] J. Ponce. On computing metric upgrades of projective reconstructions under the rectangular pixel assumption. In Second European Workshop on 3D Structure from Multiple Images of Large-Scale Environments, pages 52-67, London, UK, 2001. Springer-Verlag.

[13] J. Ponce, K. McHenry, T. Papadopoulo, M. Teillaud, and B. Triggs. On the absolute quadratic complex and its application to autocalibration. In Proc. IEEE Conference on Computer Vision and Pattern Recognition, volume 1, pages 780-787, Washington, DC, USA, 2005.

[14] T. Pribanic, P. Sturm, and S. Peharec. Wand-based calibration of 3d kinematic system. IET-CV, 3(3):124-129, September 2009.

[15] E. D. Roe, Jr. On the circular points at infinity. The American Mathematical Monthly, 4(5):132-145, May 1897.

[16] J. I. Ronda, G. Gallego, and A. Valdés. Camera autocalibration using Plücker coordinates. In International Conference on Image Processing, volume 3, pages 800-803, Genoa, Italy, 2005.

[17] J. I. Ronda, A. Valdés, and G. Gallego. Line geometry and camera autocalibration. J. Math. Imaging Vis., 32(2):193-214, 2008.

[18] Y. Seo and A. Heyden. Auto-calibration from the orthogonality constraints. In Proc. International Conference on Pattern Recognition, volume 01, pages 1067-1071, Los Alamitos, CA, USA, 2000.

[19] P. Sturm. Critical motion sequences for monocular self-calibration and uncalibrated euclidean reconstruction. Computer Vision and Pattern Recognition, IEEE Computer Society Conference on, 0:1100, 1997.

[20] P. A. Tresadern and I. D. Reid. Camera calibration from human motion. Image Vision Comput., 26(6):851-862, 2008.

[21] B. Triggs. Autocalibration and the absolute quadric. In Proc. of the IEEE Conference on Computer Vision and Pattern Recognition, pages 609-614, Puerto Rico, USA, June 1997.

[22] R. Y. Tsai. A versatile camera calibration technique for high-accuracy $3 \mathrm{~d}$ machine vision metrology using off-the-shelf tv cameras and lenses. In Radiometry, pages 221-244, USA, 1992. Jones and Bartlett Publishers, Inc.

[23] A. Valdés and J. I. Ronda. Camera autocalibration and the calibration pencil. Journal of Mathematical Imaging and Vision, 23(2):167-174, 2005.

[24] A. Valdés, J. I. Ronda, and G. Gallego. The absolute line quadric and camera autocalibration. International Journal of Computer Vision, 66(3):283-303, 2006.

[25] K.-Y. K. Wong, P. R. Mendonça, and R. Cipolla. Camera calibration from surfaces of revolution. IEEE Transactions on Pattern Analysis and Machine Intelligence, 25:147-161, 2003.

[26] H. Zhang, K.-Y. K. Wong, and G. Zhang. Camera calibration from images of spheres. IEEE Trans. Pattern Anal. Mach. Intell., 29(3):499-502, 2007.

[27] Z. Zhang. A flexible new technique for camera calibration. IEEE Transactions on Pattern Analysis and Machine Intelligence, 22:1330-1334, 2000.

[28] Z. Zhang. Camera calibration with one-dimensional objects. IEEE Transactions on Pattern Analysis and Machine Intelligence, 26:892-899, 2004. 